\title{
Recovering Stellar Population Properties and Redshifts From Broadband Photometry of Simulated Galaxies: Lessons for Sed Modeling
}

\section{Citation}

Wuyts, Stijn, Marijn Franx, Thomas J. Cox, Lars Hernquist, Philip F. Hopkins, Brant E. Robertson, and Pieter G. van Dokkum. 2009. "RECOVERING STELLAR POPULATION PROPERTIES AND REDSHIFTS FROM BROADBAND PHOTOMETRY OF SIMULATED GALAXIES: LESSONS FOR SED MODELING." The Astrophysical Journal 696 (1): 348-69. https:// doi.org/10.1088/0004-637x/696/1/348.

\section{Permanent link}

http://nrs.harvard.edu/urn-3:HUL.InstRepos:41381759

\section{Terms of Use}

This article was downloaded from Harvard University's DASH repository, and is made available under the terms and conditions applicable to Open Access Policy Articles, as set forth at http:// nrs.harvard.edu/urn-3:HUL.InstRepos:dash.current.terms-of-use\#OAP

\section{Share Your Story}

The Harvard community has made this article openly available.

Please share how this access benefits you. Submit a story.

\section{Accessibility}


Draft Version November 1, 2018

Preprint typeset using $\mathrm{I}_{\mathrm{A}}^{\mathrm{T}} \mathrm{EX}$ style emulateapj v. 11/26/03

\title{
RECOVERING STELLAR POPULATION PROPERTIES AND REDSHIFTS FROM BROAD-BAND PHOTOMETRY OF SIMULATED GALAXIES: LESSONS FOR SED MODELING
}

\author{
Stijn Wuyts ${ }^{1,2,3}$, Marijn Franx ${ }^{2}$, Thomas J. Cox ${ }^{1,3}$, Lars Hernquist ${ }^{1}$, Philip F. Hopkins ${ }^{4}$, Brant E. \\ Robertson $^{5,6,7}$, Pieter G. VAn DOKKum ${ }^{8}$ \\ Draft version November 1, 2018
}

\begin{abstract}
We present a detailed analysis of our ability to determine stellar masses, ages, reddening and extinction values, and star formation rates of high-redshift galaxies by modeling broad-band SEDs with stellar population synthesis. In order to do so, we computed synthetic optical-to-NIR SEDs for model galaxies taken from hydrodynamical merger simulations placed at redshifts $1.5 \leq z \leq 2.9$. Viewed under different angles and during different evolutionary phases, the simulations represent a wide variety of galaxy types (disks, mergers, spheroids). We show that simulated galaxies span a wide range in SEDs and color, comparable to these of observed galaxies. In all star-forming phases, dust attenuation has a large effect on colors, SEDs, and fluxes. The broad-band SEDs were then fed to a standard SED modeling procedure and resulting stellar population parameters were compared to their true values. Disk galaxies generally show a decent median correspondence between the true and estimated mass and age, but suffer from large uncertainties $\left(\Delta \log M=-0.06_{-0.13}^{+0.06}, \Delta \log a g e_{w}=+0.03_{-0.42}^{+0.19}\right)$. During the merger itself, we find larger offsets: $\Delta \log M=-0.13_{-0.14}^{+0.10}$ and $\Delta \log a g e_{w}=-0.12_{-0.26}^{+0.40} . E(B-V)$ values are generally recovered well, but the estimated total visual absorption $A_{V}$ is consistently too low, increasingly so for larger optical depths $\left(\Delta A_{V}=-0.54_{-0.46}^{+0.40}\right.$ in the merger regime). Since the largest optical depths occur during the phases of most intense star formation, it is for the highest SFRs that we find the largest underestimates $\left(\Delta \log S F R=-0.44_{-0.31}^{+0.32}\right.$ in the merger regime). The masses, ages, $E(B-V), A_{V}$, and SFR of merger remnants (spheroids) are very well reproduced.

We discuss possible biases in SED modeling results caused by mismatch between the true and template star formation history, dust distribution, metallicity variations and AGN contribution. Mismatch between the real and template star formation history, as is the case during the merging event, drives the age, and consequently mass estimate, down with respect to the true age and mass. However, the larger optical depth toward young stars during this phase reduces the effect considerably. Finally, we tested the photometric redshift code EAZY on the simulated galaxies placed at high redshift. We find a small scatter in $\Delta z /(1+z)$ of 0.031 .
\end{abstract}

Subject headings: galaxies: distances and redshifts - galaxies: high redshift - galaxies: ISM - galaxies: stellar content

\section{INTRODUCTION}

Understanding the growth and aging of galaxies over cosmic time requires reliable estimates of their mass, formation epoch and star formation history. With the current generation of telescopes, stellar velocity dispersion measurements can probe the gravitational potential in which the baryonic galaxy content resides out to $z \sim 1.3$ (van Dokkum \& Stanford 2003; Holden et al. 2005). Beyond this redshift, gas velocity dispersions can be measured from emission lines, but do not always trace the potential due to outflows (Franx et al. 1997; Pettini et al. 1998, 2001; Shapley et al. 2003), and would lead to

\footnotetext{
Electronic address: swuyts@cfa.harvard.edu

1 Harvard-Smithsonian Center for Astrophysics, 60 Garden Street, Cambridge, MA 02138

2 Leiden University, Leiden Observatory, P.O. Box 9513, NL2300 RA, Leiden, The Netherlands.

3 W. M. Keck Postdoctoral Fellow

4 Department of Astronomy, University of California Berkeley, Berkeley, CA 94720

5 Kavli Institute for Cosmological Physics, and Department of Astronomy and Astrophysics, University of Chicago, Chicago, IL 60637

6 Enrico Fermi Institute, Chicago, IL 60637

7 Spitzer Fellow

8 Department of Astronomy, Yale University, New Haven, CT 06520-8101
}

biased samples missing quiescent galaxies lacking emission lines in their spectra (Kriek et al. 2006). For these reasons, most studies of high-redshift galaxies have used stellar mass estimates derived by modeling of the broadband stellar energy distribution (SED) to characterize the mass.

Since age estimates from $H \alpha$ equivalent widths (van

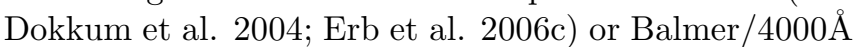
break strengths (Kriek et al. 2006) are very demanding in terms of telescope time and only attainable for the brightest galaxies, stellar ages as well are commonly derived from broad-band photometry.

Over the past few years, SED modeling has been proven extremely valuable in characterizing the galaxy population in the early universe (e.g., Papovich et al. 2001; Shapley et al. 2001, 2005; Förster Schreiber et al. 2004). Nevertheless, a number of assumptions are required for the limited number of data points (11 passbands in our case, but often less) to lead to a single solution in terms of physical properties such as stellar mass, stellar age, dust extinction, star formation rate (SFR), and often redshift.

First, the star formation history ( $\mathrm{SFH}$ ) is generally modelled by a simple functional form: a single burst, constant star formation, or an exponentially declining 
model. In reality, high-redshift galaxies show evidence of more complex SFHs, often with brief recurrent episodes of star formation (e.g. Papovich et al. 2001; Ferguson et al. 2002; Papovich et al. 2005). Second, we use the approximation of a single foreground screen of dust in accounting for the attenuation, even though in reality the dust will be distributed in between the stars. Third, we fit solar metallicity models. This is a common approach in modeling of high-redshift galaxy SEDs in order to reduce the number of degrees of freedom in the fitting procedure by one (e.g., Förster Schreiber et al. 2004; Shapley et al. 2005; Rigopoulou et al. 2006). In addition, the tracks and spectral libraries on which the stellar population synthesis models are based, have the best empirical calibration for solar metallicity. Although consistent with the current metallicity estimates from near-infrared (NIR) spectroscopy of high-redshift galaxies (van Dokkum et al. 2004; Erb et al. 2006a; Maiolino et al. 2008), it must be kept in mind that these measurements are currently limited to the bright end of the galaxy population. Fourth, SED modeling generally assumes a purely stellar origin of the light, while observational evidence for a substantial fraction of low luminosity AGN at high redshift has been accumulating (van Dokkum et al. 2004; Reddy et al. 2005; Papovich et al. 2006; Kriek et al. 2007; Daddi et al. 2007b). They may contribute to the optical SEDs.

Finally, one adopts a certain attenuation law, initial mass function (IMF), and stellar population synthesis code. Their appropriateness at low and high redshifts is much debated.

In this paper, we address the impact of the first four assumptions (related to SFH, dust attenuation, metallicity, and AGN) using hydrodynamical simulations of merging galaxies (see Robertson et al. 2006; Cox et al. 2006). The SPH simulations follow the star formation on a physical basis, resulting in more complex SFHs than are allowed in typical SED modeling. They keep track of the distribution and metallicity of gas and stellar particles, allowing a determination of the line-of-sight dependent extinction toward each stellar particle separately and a knowledge of the stellar metallicity as a function of time. Here, we apply the same SED modeling that we use for observed galaxies to broad-band photometry extracted from the simulation outputs, and study how well the mass, age, dust content, and SFR of the simulated galaxies can be recovered.

The reason we use merger simulations for this exercise is threefold. First, galaxy mergers are believed to play an important role in galaxy evolution (see, e.g., Holmberg 1941; Zwicky 1956; Toomre \& Toomre 1972; Toomre 1977), increasingly so at high redshift (see, e.g., Glazebrook et al. 1995; Driver, Windhorst,\& Griffiths 1995; Abraham et al. 1996). Moreover, along their evolutionary path they are visible as vastly different galaxy types, allowing to test the recovery of stellar population parameters under a wide range of conditions: gas-rich star-forming disks, dust-obscured mergers, and quiescent spheroids. Finally, in a separate paper we will compare predictions of the color distribution and mass density of high-redshift galaxies derived from these simulations with the observed galaxy population in deep fields. A good understanding of what it is we measure with SED modeling is crucial in order to compare identical mass- limited samples of observed and simulated galaxies.

We start with a description of the simulations in $\S 2$. Next, we explain the methodology of our SED modeling in $\S 3$. $\S 4$ discusses how well we can measure stellar population properties when a spectroscopic redshift is available. $§ 5$ repeats the analysis, now leaving the redshift as an extra free parameter (i.e., fitting for the photometric redshift). Finally, we summarize the results in $\S 6$.

\section{THE SIMULATIONS}

\subsection{Main characteristics}

The simulations on which we test our SED modeling were performed by Robertson et al. (2006). We refer the reader to that paper for a detailed description of the simulations. Briefly, the simulations were performed with the parallel TreeSPH code GADGET-2 (Springel 2005). The code uses an entropy-conserving formulation of smoothed particle hydrodynamics (Springel \& Hernquist 2002), and includes gas cooling, a multiphase model for the interstellar medium (ISM) to describe star formation and supernova feedback (Springel \& Hernquist 2003), and a prescription for supermassive black hole growth and feedback (Springel et al. 2005b).

At the start, each simulation consists of 120000 dark matter particles, 80000 gas particles, and 80000 stellar particles. They represent two stable, coplanar disk galaxies, each embedded in an extended dark matter halo with Hernquist (1990) profile. We have realisations where the disks start with a gas fraction of $40 \%$ and $80 \%$. Stellar masses at the start of the simulation varied from $7.0 \times 10^{9} M_{\odot}$ to $2.3 \times 10^{11} M_{\odot}$ per disk galaxy. In total, we study 6 simulations (2 different gas fractions, and 3 different masses) for which at 30 timesteps all information was stored in a snapshot. Each snapshot was analyzed as seen from 30 different viewing angles and placed at 8 redshifts between $z=1.5$ and $3(\S 2.2)$. We repeated our analysis on a limited number of snapshots and viewing angles of merger simulations of non-coplanar disks and conclude that the results presented in this paper are robust against such variations.

For a given virial velocity, the halo concentration, virial mass and virial radius were scaled following Robertson et al. (2006) to approximate the structure of disk galaxies at redshift $z=3$. In practice, this means that the massand redshift-dependent halo concentration measured by Bullock et al. (2001) was adopted:

$$
C_{v i r}\left(M_{v i r}, z\right) \approx 9\left(\frac{M_{v i r}}{M_{\text {coll }, 0}}\right)^{-0.13}(1+z)^{-1}
$$

where $M_{\text {coll }, 0} \sim 8 \times 10^{12} h^{-1} M_{\odot}$ is the linear collapse mass at $\mathrm{z}=0$, and that the following scaling relations were used for the virial mass and virial radius of the progenitors:

$$
\begin{gathered}
M_{v i r}=\frac{V_{v i r}^{3}}{10 G H(z)} \\
R_{v i r}=\frac{V_{v i r}}{10 H(z)},
\end{gathered}
$$

where $V_{v i r}$ is the virial velocity and $H(z)$ is the Hubble parameter. Disk sizes were initialized according to the Mo et al. (1998) formalism for dissipational disk galaxy formation assuming the fraction of the total angular momentum contained in the disk equals the fraction of the 
total mass contained in the disk $\left(\frac{M_{\text {disk }}}{M_{v i r}}=0.041\right.$ to match the Milky Way-like model by Springel et al. 2005a). The disk scale length is then derived from the halo concentration $C_{\text {vir }}$ (Eq. 1) and the galaxy spin $\lambda$. The adopted value of $\lambda=0.033$ is motivated by cosmological N-body simulations (Vitvitska et al. 2002).

We set the ages of the stars existing at the start of the simulation such as to represent a constant star formation history prior to the start of the simulation at a star formation rate (SFR) equal to that calculated in the first phases of the simulation. The corresponding metallicities of stars present at the start of the simulation were then set according to the closed box model: $Z(t)=-y \ln \left[f_{\text {gas }}(t)\right]$, where $Z(t)$ is the metallicity of a stellar particle formed at time $t$, the yield $y=0.02$ and $f_{\text {gas }}(t)$ is the gas fraction of the system at the considered time. Similarly, the gas at the start of the simulation was assigned a uniform metallicity $Z_{g a s}\left(t_{S}\right)=-y \ln \left[f_{\text {gas }}\left(t_{S}\right)\right]$ where $t_{S}$ represents the start of the simulation, and $f_{\text {gas }}\left(t_{S}\right)=0.4$ or 0.8 respectively for our 2 gas fraction runs. The closed box model represents an upper limit on the allowed enrichment by heavy elements, which in reality may be reduced by outflows or infall of metalpoor gas (Edmunds 1990). The fact that we consider 2 gas fractions guarantees a wide range of progenitor types, with ages of a few $100 \mathrm{Myr}$ and $Z_{\text {gas }}=0.004$ at the start of the simulation for $f_{\text {gas }}=0.8$ to typical stellar ages of a Gyr and nearly solar gas metallicity for $f_{\text {gas }}=0.4$. Starting from the initial conditions described above, the GADGET-2 code computed the subsequent evolution of the stellar populations using the star formation and metal enrichment prescriptions outlined by Springel \& Hernquist (2003). The mass-weighted mean stellar metallicity for the $f_{\text {gas }}=0.4$ runs increases in $\sim 700 \mathrm{Myr}$ from $0.4 Z_{\odot}$ to $0.9 Z_{\odot}$, after which it remains constant. The metal enrichment of stars in the $f_{\text {gas }}=0.8$ runs proceeds rapidly, increasing in a few 100 Myr from $0.1 Z_{\odot}$ to $0.5 Z_{\odot}$ and leveling off after $\sim 0.5$ Gyr at values between $0.8 Z_{\odot}$ and $Z_{\odot}$.

The overall timespan covered by each simulation was 2 Gyr. Fig. 1(a) illustrates the star formation history of one of the merger simulations. Specifically, we show a simulation starting with $40 \%$ gas fraction and ending with a stellar mass of $1.5 \times 10^{11} M_{\odot}$, but the SFH of the other simulations looks qualitatively similar. Fig. 1(b) illustrates the build-up of stellar mass. In order to allow a fair test of the SED modeling with Bruzual \& Charlot (2003) templates (that include mass loss), we take into account mass loss of each of the stellar particles for which the time of formation and gas mass from which it was formed are stored in the simulation snapshots. This explains the slow decrease in total stellar mass at late times in Fig. 1(b). Fig. 1(c) shows the exhaustion of gas from which the stars are formed, and Fig. 1(d) presents the accretion history onto the black hole(s). We draw the time axis relative to the actual moment of merging, defined as the timestep when the two black hole particles become one, coinciding with the peak in the accretion history. Cross symbols indicate the snapshots, separated by $70 \mathrm{Myr}$, when all physical information was stored to disk.

As time progresses, the orderly rotation and star formation in the disks is disturbed by each others gravi-
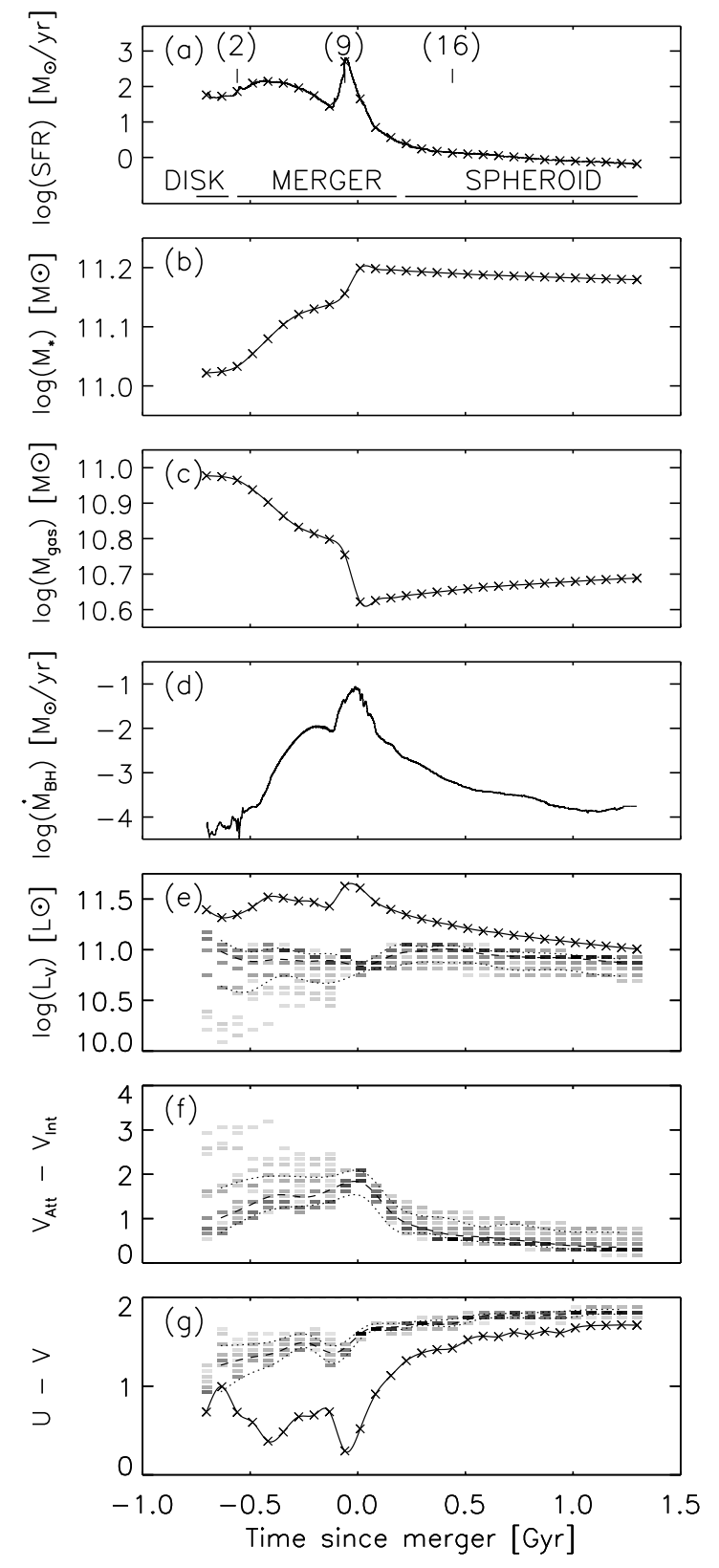

FIG. 1.- Evolution of a typical merger simulation. (a) The star formation history, (b) the mass build-up, (c) the gas exhaustion, (d) the accretion rate history onto the black hole(s), (e) the evolution of the intrinsic (i.e., unattenuated) and attenuated Vband luminosity, (f) the distribution of effective visual extinctions (attenuated minus intrinsic V-band magnitude) corresponding to different viewing angles, and $(\mathrm{g})$ the intrinsic and attenuated $U-V$ color. Cross symbols in panel (e) and (g) present the evolution of the intrinsic photometry. For the attenuated photometry in panels (e) to $(\mathrm{g})$, a binned representation is used where a darker intensity indicates a larger number of viewing angles. The dashed curve represents the median evolution. The dotted lines indicate the interval containing the central $68 \%$ of the viewing angles. The cross symbols mark the sampling of snapshots when the full physical information of all SPH particles was stored to disk. After a first bump in the star formation rate during the first passage of the progenitors, a peak in star formation is reached for a brief period during which several hundreds of solar masses of gas are converted into stars. The typical extinction for a random line of sight is peaking around the same time. Shortly after, the accretion onto the supermassive black hole is maximal, coinciding with the merger between the two progenitor black holes. The reddest U-V colors are reached during the merger remnant phase. 
tational pull. The star formation history shows a first, but rather shallow, bump during the first passage of the disks. Next, gravitational torques enable the gas to loose angular momentum and flow to the centers where it triggers a starburst (Larson \& Tinsley 1978; Noguchi 1988; Hernquist 1989; Barnes \& Hernquist 1991, 1996; Mihos \& Hernquist 1994, 1996). Meanwhile, part of the inflowing gas is fed to the central supermassive black holes (SMBHs). Once the SMBHs grow massive enough, they produce a luminous quasar (Sanders et al. 1988a,b; Hernquist 1989; Sanders \& Mirabel 1996; Genzel et al. 1998) whose feedback contributes to halting subsequent star formation (Di Matteo et al. 2005; Springel et al 2005a), leaving a red spheroid galaxy as remnant (Robertson et al. 2006; Cox et al. 2006).

\subsection{Extracting photometry from the simulation output}

The evolutionary path as outlined in $\S 2.1$ is followed by the GADGET- 2 code at a fine time resolution $\left(\Delta t \sim 10^{4}\right.$ yr). At sparser timesteps (70 Myr apart), the positions, masses, ages, and metallicities of all particles were stored. It is from these simulation snapshots that we derive the observed SEDs of the merger as a function of time. The applied technique is similar to that used by Robertson et al. (2007) to translate simulations of the most massive $z \sim 4-14$ galaxies into observables.

The light a virtual observer would receive from the simulated merger, is composed of stellar and AGN emission, the latter only contributing significantly during a brief period of time. We ignore any contribution from emission lines produced by the gas content of the galaxies, possibly contributing on the order of $0.1 \mathrm{mag}$ in the optical broad-band photometry. Furthermore, we account for attenuation by interstellar dust and Lyman forest attenuation by the intervening medium between the redshifted galaxy and the observer following Madau (1995). The combination of these steps, described in this section, leads to observables that are similar to the real observations that we model with stellar population synthesis codes.

First, we focus on the computation of intrinsic (i.e., unattenuated) magnitudes from the stellar component. Each of the stellar particles is treated as a single stellar population characterized by its mass, age, and metallicity. We choose to use the Salpeter (1955) IMF, as was done in previous observational work (e.g. Förster Schreiber et al. 2004; Wuyts et al. 2007). We then interpolate the corresponding luminosity for each stellar particle from a grid of SSP templates with different ages and metallicities from the stellar population synthesis code by Bruzual \& Charlot (2003, hereafter BC03). Fig. 1 (e) illustrates the evolution of the intrinsic rest-frame $V$-band luminosity for one of the simulations.

For the AGN emission, we scale a luminositydependent template SED by the bolometric black hole luminosity given by the simulation. The template SED was derived from the optically blue (i.e., unreddened) quasar sample by Richards et al. (2006) with locally attenuated light being reprocessed as an IR bump longward of $\lambda>1 \mu \mathrm{m}$. A full discussion of the AGN template is presented by Hopkins, Richards,\& Hernquist (2007). In most of our analysis, we will consider the stellar light only. $\S 4.5$ addresses the impact AGN can have on the outcome of SED modeling during the brief period when its contribution to the total light is significant.

Galaxies, certainly in their actively star-forming phases, are not devoid of gas and dust. It is therefore crucial to account for the obscuring and reddening effect dust has on the stellar and AGN emission. We compute the optical depth along the line of sight toward each stellar particle. To do so, we compute the local gas density on a fine grid derived from the SPH formalism and the particle distribution (Hopkins et al. 2005a) and integrate out from each particle along the line of sight to large distance. The simulations are based on the GADGET multi-phase ISM model developed by Springel \& Hernquist (2003). This model calculates the local mass fraction in the warm/hot $\left(T=10^{5}-10^{7} \mathrm{~K}\right.$, diffuse, partially ionized) and cold $\left(T=10^{3} \mathrm{~K}\right.$, in the simulations both associated with molecular clouds and HI cloud cores) phases of dense gas, assuming pressure equilibrium between the two phases. Following Hopkins et al. (2005b), the attenuation along the line of sight is then derived from the density of the warm/hot-phase component only. Hopkins et al. (2005b) found typically small volume filling factors $(<0.01)$ and cross sections of the cold-phase "clumps", motivating their approach. The assumption that most of the lines of sight only pass through the warm/hot-phase component provides effectively a lower limit on the optical depths. We use a gas-to-dust ratio equal to that of the Milky Way, $\left(A_{B} / N_{\mathrm{HI}}\right)_{\mathrm{MW}}=8.47 \times 10^{-22} \mathrm{~cm}^{2}$, with a linear scaling factor accounting for gas metallicities deviating from solar: $A_{B} / N_{\mathrm{HI}}=(Z / 0.02)\left(A_{B} / N_{\mathrm{HI}}\right)_{\mathrm{MW}}$. As default, we adopt the Calzetti et al. (2000) attenuation law for the wavelength dependence of the optical depth. Changes in the synthetic photometry when adopting a SMC-like or Milky Way-like attenuation law from Pei (1992) will be discussed. The computation of optical depths was repeated for 30 viewing angles, with directions uniformly spaced in solid angle $d \cos \theta d \phi$. Fig. 1(f) presents the distribution of effective visual extinction values (observed minus intrinsic V-band magnitude) as a function of time since the merger. The extinction varies in the following way. In the early stages typical extinction values are modest, with the exception for a few lines-of-sights were the disks are seen edge-on. The overall extinction along all lines-of-sight reaches a peak during the mergertriggered starburst and drops to very low values after star formation has ceased.

Finally, in computing the observer-frame apparent magnitudes, we redshift the attenuated SED and convolve it with the same set of filtercurves that we have deep observations for in the Chandra Deep Field South (CDFS; Wuyts et al. 2008): $B_{435}, V_{606}, i_{775}, z_{850}, J$, $H, K_{s},[3.6 \mu \mathrm{m}],[4.5 \mu \mathrm{m}],[5.8 \mu \mathrm{m}]$, and $[8.0 \mu \mathrm{m}]$. Here, we apply the depression factors $D_{A}(z)$ and $D_{B}(z)$ given by Madau (1995) for the Lyman forest attenuation of the continuum between $\operatorname{Ly} \alpha$ and $\operatorname{Ly} \beta$ and between $\operatorname{Ly} \beta$ and the Lyman limit respectively. The flux blueward of the Lyman limit $\left(\lambda_{L}=912 \AA\right)$ was set to 0 , as is done by the HYPERZ code (v1.1, Bolzonella et al. 2000) that we use for SED modeling.

In practice, it is computationally more convenient to interpolate the apparent magnitudes in a given passband for each of the stellar particles on a precompiled grid of BC03 apparent magnitudes at the redshift of interest. The internal dust attenuation is then applied using the 
value of the Calzetti et al. (2000) attenuation law at the effective wavelength for that passband. We tested that this method, as opposed to attenuating the full resolution BC03 spectrum and then convolving with the filtercurve, leads to photometric differences of at most a few percent.

We note that we never attempt to separate the light into the contribution from the two progenitors. Instead, we always study the total photometry, as if the merging system were unresolved.

In future studies, we will employ full-fledged radiative transfer codes such as SUNRISE (Jonsson 2006) to translate the simulation snapshot information to observables. SUNRISE calculates scattering, absorption, and reemission of light passing through the cold and/or hot phase of the ISM, with the optional inclusion of a subgrid model that accounts for the obscuring effects of birth clouds in which young star-forming regions are embedded (Groves et al. 2008). Reemission by dust only influences the spectral shape longward of the wavelength bands used in our SED modeling. Preliminary analysis of SUNRISE SEDs indicates that the other effects might lead to a stronger extinction during the star-forming phases, while leaving the spheroid photometry unchanged with respect to the photometry computed in this paper. It will be interesting, in the light of the findings presented here, to investigate the shape of the effective attenuation law obtained with such a more sophisticated code, and its dependence on the parameters of the radiative transfer. For the sake of this paper, our line-of-sight photometry has the advantage of being transparent and simple.

\subsection{The color evolution of merging galaxies}

With the synthetic photometry at hand, we are now able to follow the color evolution of simulated galaxies throughout the merger event. We illustrate the spatially resolved color evolution for a face-on view of a typical simulation in Fig. 2. This particular simulation has an initial gas fraction of $80 \%$ and final stellar mass of $1.2 \times 10^{11} M_{\odot}$. The 3-color images are composed of rest-frame $U$ (blue), $V$ (green), and $J$ (red). On each postage stamp, we indicate the snapshot number, time since the merger, and whether the intrinsic (Int) or attenuated (Att) colors are plotted. The interval between consecutive snapshots is $70 \mathrm{Myr}$. The color distribution is clearly not homogeneous throughout the merger evolution. Instead, color gradients are present, with the nuclear regions being intrinsically bluer due to new star formation, but effectively redder than the surrounding material due to the presence of dust. We return to this point in an analysis of observed versus simulated galaxy colors by Wuyts et al. (2009b). In this paper, we restrict ourselves to the study of integrated colors.

The corresponding evolutionary tracks in integrated rest-frame $U-V$ versus $V-J$ color-color space are presented in Fig. 3. Labbé et al. (2005) first introduced the observed-frame equivalent of this diagram to illustrate the wide range of galaxy types at high redshift ranging from blue, relatively unobscured star-forming systems to dusty starbursts to quiescent red galaxies. The color criterion proposed by Labbé et al. (in preparation) to select galaxies of quiescent nature is plotted as the red wedge in Fig. 3.

Considering the intrinsic (unattenuated) colors, the evolutionary track has a direct relation to the mean light- weighted age (and depends to a lesser degree on the gradual increase in stellar metallicities). It bends down to bluer $U-V$ as new star formation is triggered during first passage of the progenitors, and again during the shorterlived nuclear starburst phase. Eventually, the color track leads to the region in color-color space where also quiescent observed galaxies reside. In reality, the path taken in color-color space will not merely depend on the star formation history. Taking into account attenuation by dust, the integrated colors get redder. This is particularly true during the phases of active star formation. We note that the precise shape of the color track depends not only on initial conditions (gas fraction, mass, disk orientations, ...) but also on viewing angle. In fact, as illustrated in Fig. 3, color differences between different viewing angles at a given time can be of similar size as color changes between different times for a given viewing angle. At later times, the role of dust is minimal and the remnant converges to colors typical for quiescent galaxies (i.e., within the red wedge).

\subsection{The colors and SEDs of simulated and observed galaxies}

Prior to analyzing the performance of our SED modeling procedure, it is important to confirm that the simulated galaxies have spectral shapes resembling those of real high-redshift galaxies in observed deep fields, thus validating their role as test objects. To this end, we indicate the binned color distribution of simulated galaxies, viewed from different angles and during different phases of their evolution, in a rest-frame $U-V$ versus $V-J$ color-color diagram (Fig. 4). Plus symbols show the location of observed galaxies in the HDFS (Labbé et al. 2003), MS 1054-03 (Förster Schreiber et al. 2006), and the CDFS (Wuyts et al. 2008) selected by their photometric redshift (or spectroscopic when available) to lie in the same redshift range $(1.5<z<3.0)$. We also applied a stellar mass cut at $M_{*}>1.4 \times 10^{10} M_{\odot}$ for the observed sample; the lowest initial stellar mass for the considered set of simulations. Here, we do not attempt to statistically compare the two samples. The abundances of different types of galaxies as predicted from the simulations will be addressed by Wuyts et al. (2009b). For our current purpose of analyzing the effects of star formation history, dust, metallicity and AGN on SED modeling, it is sufficient to note that there is a large overlap between the color-color space spanned by the simulated and observed galaxies. Every simulated galaxy considered in this paper has counterparts in the real universe with similar rest-frame optical and optical-to-NIR colors. However, the observed distribution extends to colors that are redder by a few $0.1 \mathrm{mag}$, both in $U-V$ and in $V-J$, than the simulations considered here. Given the one-sided nature of the different color spread, it is unlikely that this can be attributed to photometric uncertainties alone. Therefore, we caution that our results may not necessarily be extrapolated to the reddest galaxies present in observed samples. Wuyts et al. (2009b) discuss several possible origins of the color discrepancy, particularly in $V-J$, ranging from the method to compute model photometry to differences between the evolutionary history of highredshift galaxies in the real universe and in our merger simulations. There we analyze, for example, the presence of color gradients, and the dependence of the color 

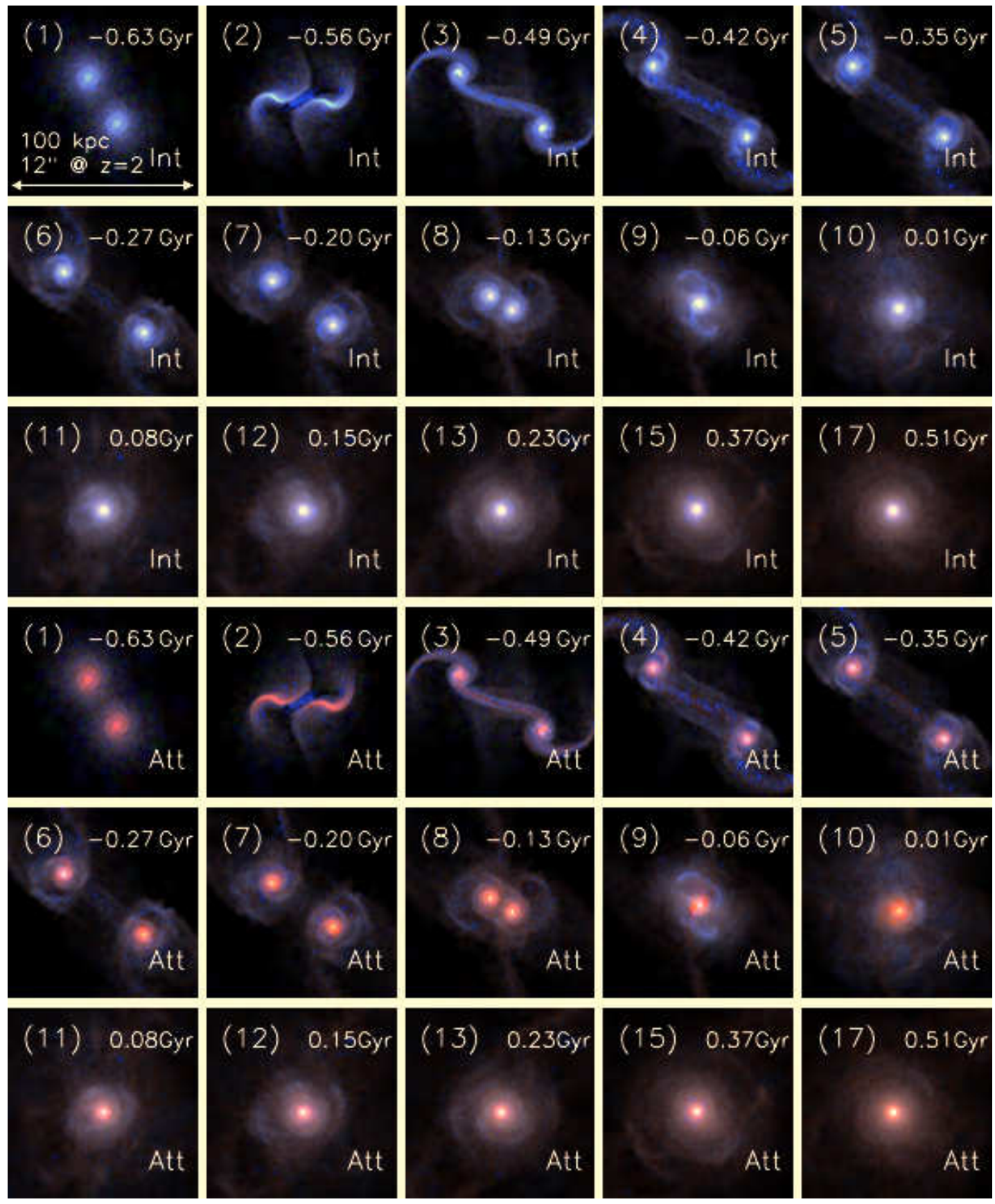

FIG. 2.- Three-color postage stamps (rest-frame $U$ (blue), $V$ (green), $J(r e d)$ ) of a typical merger simulation. The top three rows illustrate the intrinsic evolution, ignoring extinction. The bottom three rows show the evolution when taking into account attenuation by dust. Significant changes in color are observed over time, and between intrinsic and attenuated images.

distribution on the adopted attenuation law and stellar population synthesis code. Other models than BC03 that include a more significant contribution of the thermally pulsing asymptotic giant branch (particularly in the near-IR) result in a color distribution that is shifted towards redder $V-J$ colors (Maraston 2005; Maraston et al. 2006).

To ascertain that observed and simulated galaxies with similar $U-V$ and $V-J$ colors have similar SEDs over the whole spectral range, Fig. 5 presents the rest-frame SEDs of objects in region 1-6 of Fig. 4. Again, the binned distribution represents the simulations, with darker grayscales 


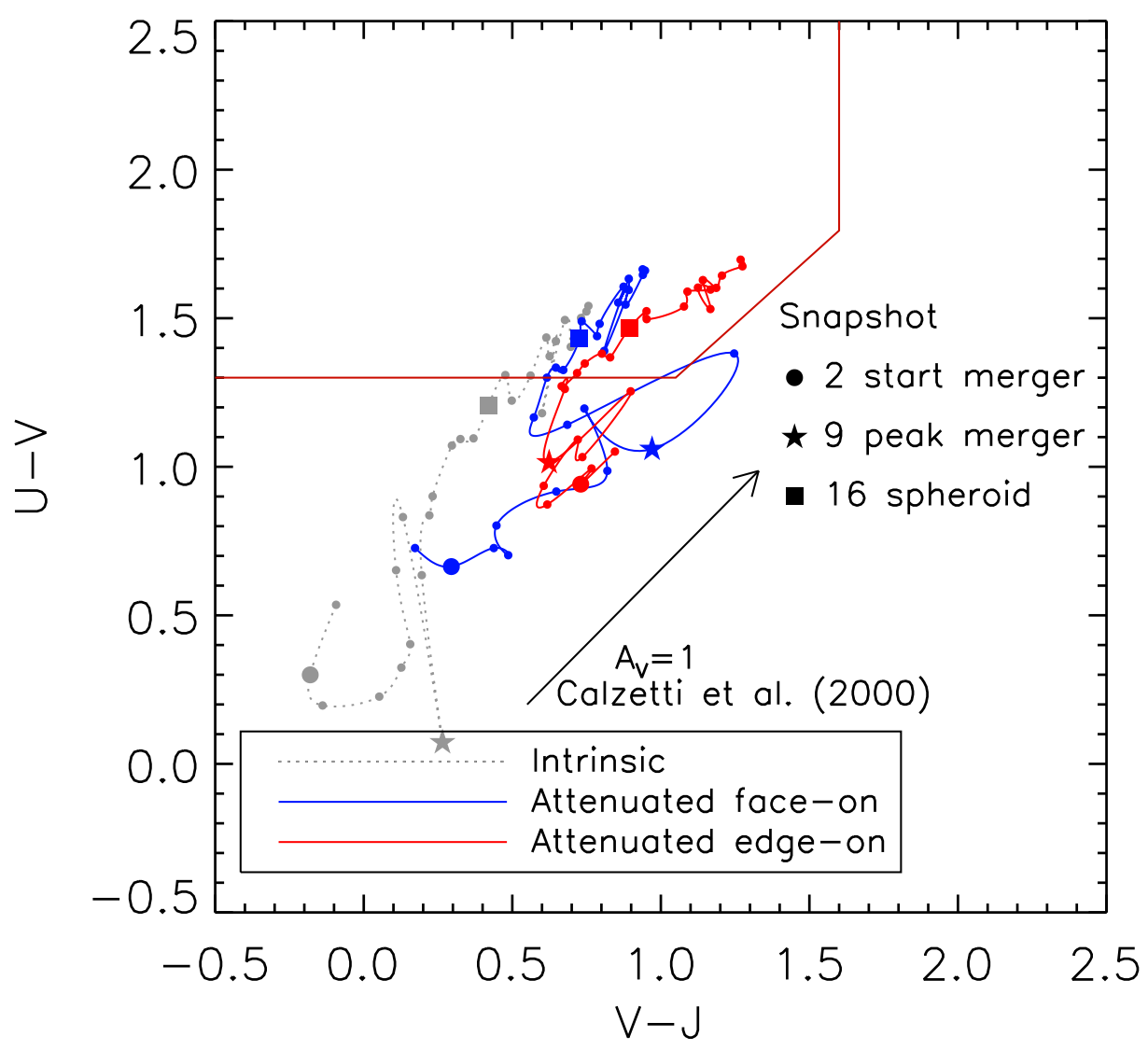

Fig. 3.- Evolutionary tracks in a rest-frame $U-V$ versus $V-J$ color-color diagram for the typical merger simulation shown in Fig. 2. We illustrate the intrinsic color evolution as well as the evolution of attenuated colors with time as we view the merging pair face- and edge-on. Snapshots 70 Myr apart are marked with small dots. Key phases corresponding to the numbers indicated in Fig. 2 and Fig. 14 are indicated with large symbols. Time since (or before) then actual moment of merging is indicated in the top right corner of each panel. Colors can vary significantly at a given time (by $\sim 0.5 \mathrm{mag}$ ) as we view the merging system from different lines of sight. The starburst occuring during the merger is very dusty, leading to redder colors at that stage.

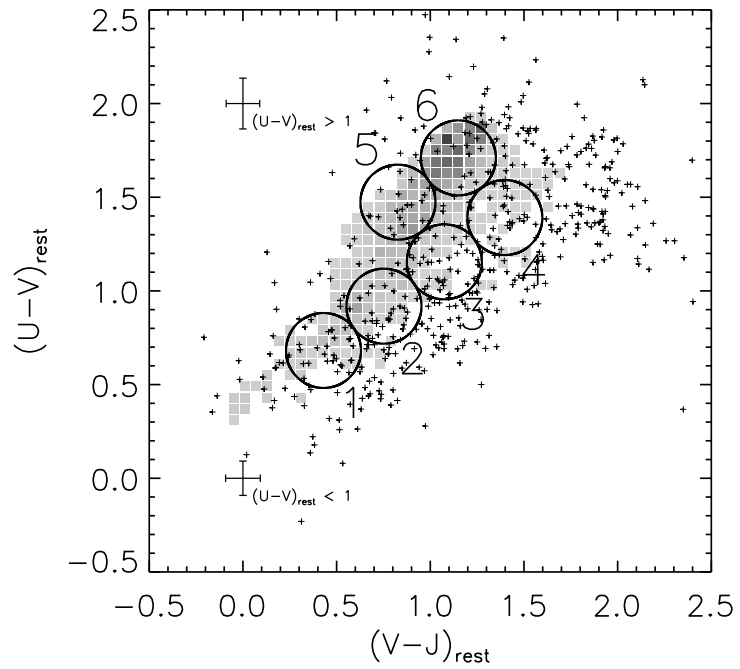

Fig. 4.- Rest-frame $U-V$ versus $V-J$ color-color diagram showing the binned color distribution of the simulations seen under different viewing angles and at different epochs. Overplotted (plus symbols) are the rest-frame colors of observed galaxies with $M_{*}>$ $1.4 \times 10^{10} M_{\odot}$ at $1.5<z<3$ in the HDFS, MS 1054-03, and the CDFS. Observed galaxies with matching colors are found for all simulated galaxies. The reddest observed sources in $U-V$ and $V-J$ are not reproduced by the considered set of simulations. Rest-frame SEDs for sources in regions 1-6 are displayed in Fig. 5. indicating a larger number of objects. Overplotted with black dots is the broad-band photometry of our observed sample within the same region of color-color space, placed at the respective rest-frame wavelength. The SEDs are normalized to the rest-frame $V$-band. By selection, the observed and simulated photometry matches well at rest-frame $U$ and $J$. In between the $U V J$ filters, and outside the U-to-J range, no correspondence was imposed. The fact that the UV spectral shape and the NIR tail of the observed and simulated SEDs show a general agreement, is encouraging. We conclude that the simulated photometry can be adopted as a realistic input to our SED modeling procedure. The results of our analysis will be applicable to observed galaxies with similar colors.

\section{SED MODELING: METHODOLOGY}

We characterize physical parameters such as stellar mass, stellar age, dust attenuation, and SFR by matching the observed-frame broad-band photometry to synthetic templates from the stellar population synthesis code by BC03. We use the HYPERZ stellar population fitting code, version 1.1 (Bolzonella et al. 2000) and fit the SED twice: first fixing the redshift to the true value (for which we computed the simulated photometry), next adopting a photometric redshift estimate obtained from the EAZY version 1.0 photometric redshift code (Brammer 


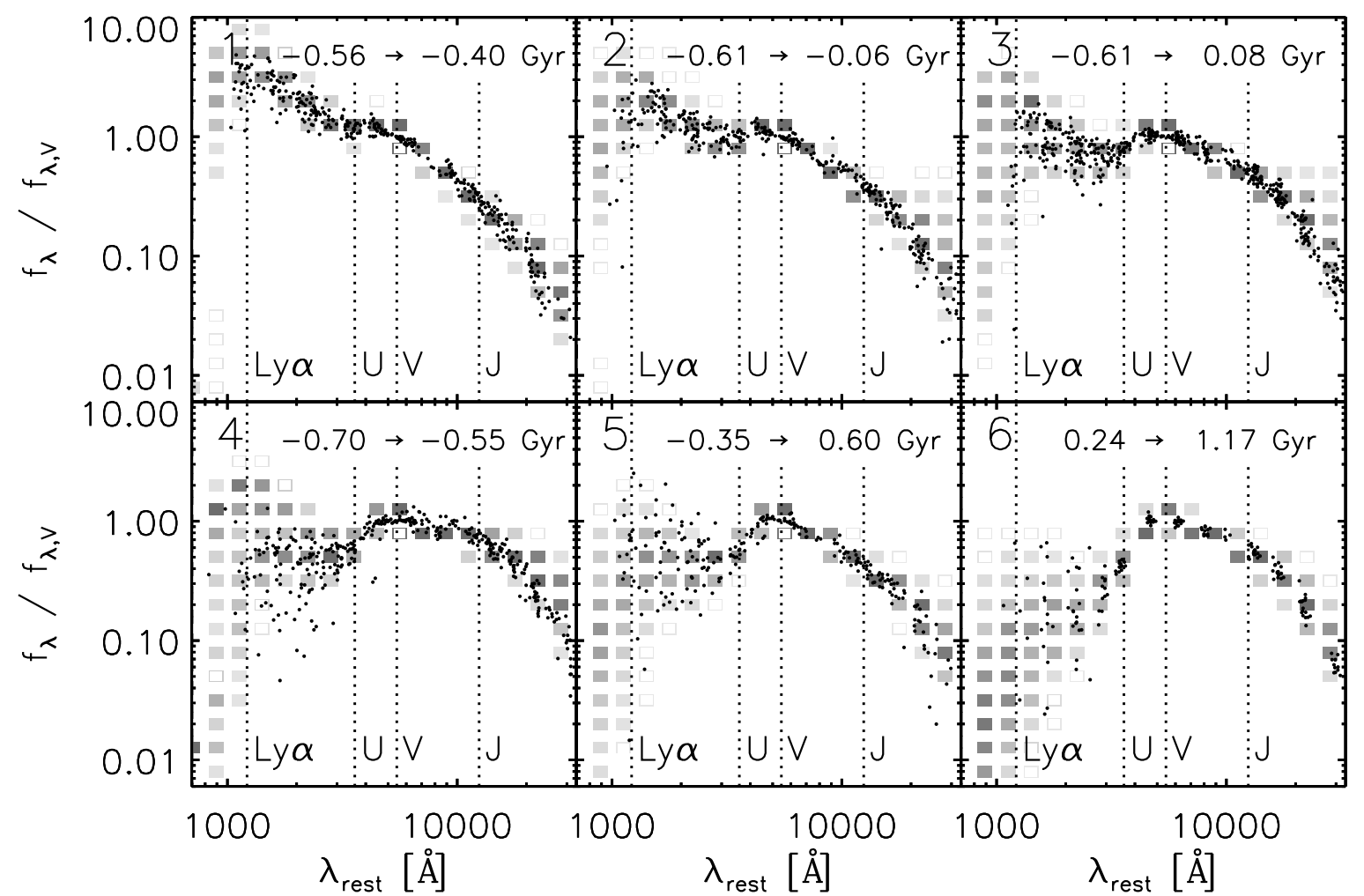

FIG. 5.- Rest-frame SEDs of simulated galaxies in regions 1-6 of Fig. 4. A darker intensity of the binned representation indicates a larger density of simulated galaxies with that flux level. In each panel, we give the central $68 \%$ interval of the distribution of times before or since the merger for the simulation snapshots with photometry in the respective region. Overplotted (black dots) are the rest-frame broad-band SEDs of observed $1.5<z<3$ galaxies with $M_{*}>1.4 \times 10^{10} M_{\odot}$ in the HDFS, MS 1054-03, and the CDFS. A general agreement between observed and simulated spectral shapes is observed, also outside the $U$-to- $J$ range where the correspondence was not imposed by selection.

et al. in preparation). In each case, the full $B_{435}$-to- 8 $\mu \mathrm{m}$ SED, sampled with identical passbands as available for the GOODS-CDFS $\left(B_{435}, V_{606}, i_{775}, z_{850}, J, H, K_{s}\right.$, $[3.6 \mu \mathrm{m}],[4.5 \mu \mathrm{m}],[5.8 \mu \mathrm{m}],[8.0 \mu \mathrm{m}])$, was fed to $\mathrm{HY}-$ PERZ. Random photometric uncertainties were assigned as to mimic real observations in the CDFS, and fluxes in each band were perturbed accordingly. Precisely, for each of the 43200 SEDs corresponding to a simulated galaxy observed during a certain phase of its evolution, placed at a certain redshift, and observed along a certain line-of-sight, we compute 5 realizations of the SED by introducing a gaussian perturbation in all bands with the amplitude derived from the depth of GOODS-CDFS observations in the respective bands. A minimum error of 0.08 mag was adopted for all bands, preventing small errors from dominating the fit.

As in Wuyts et al. (2007), we selected the least $\chi^{2}$ solution out of three possible star formation histories: a single stellar population (SSP) without dust, a constant star formation (CSF) history with dust ( $A_{V}$ varying from 0 to 4 in steps of 0.2 ), and an exponentially declining star formation history with a fixed $e$-folding timescale of $300 \mathrm{Myr}\left(\tau_{300}\right)$ and identical range of $A_{V}$ values. Ages were constrained to be larger than $50 \mathrm{Myr}$, to prevent improbably young ages, and smaller than the age of the universe at the observed redshift. We used a Calzetti et al. (2000) attenuation law, and assumed solar metallicity and a Salpeter (1955) IMF with lower and upper mass cut-offs $0.1 M_{\odot}$ and $100 M_{\odot}$. In recent literature, several authors have used an IMF with fewer low mass stars, such as presented by Kroupa (2001). We note that the choice of IMF is not the focus of this study, and our results remain valid as long as we use the same IMF to compute the synthetic photometry as to model the resulting SEDs. Likewise, we use for consistency the same stellar population synthesis code (BC03) to compute and to fit the synthetic SEDs.

When refering to the age derived from SED modeling, we mean the mass-weighted age obtained by integrating over the different ages of SSPs that build up the best-fit SFH, weighted with their mass fraction taking into account mass loss over time. This measure aims to quantify the age of the bulk of the stars. For an SSP, it equals the time passed since the single burst. For a CSF history, it is essentially half the time passed since the onset of star formation. The $\tau_{300} \mathrm{SFH}$ represents an intermediate case.

\section{RESULTS FROM SED MODELING AT FIXED REDSHIFT}

First, in $\S 4.1$, we present the overall performance of the standard SED modeling applied to the 'full' photometry, taking into account the effects of both attenuation, metallicity, and AGN contribution as realistically as possible. Second, we discuss the impact from different aspects influencing the colors and luminosities of galaxies separately. In order to isolate effects from star formation history ( $\$ 4.2)$, dust attenuation $(\S 4.3)$, metallicity variations ( $\$ 4.4)$, and AGN contribution ( $\$ 4.5)$, we computed the photometry for each snapshot with and without at- 
tenuation, with and without AGN contribution, and using solar metallicity, or the metallicity as computed by the simulation for each stellar particle. To each of these sets of SEDs, we applied the modeling described in $\S 3$. This approach enables us to reconstruct the analysis step by step, adding one aspect at a time. Finally, we discuss what our analysis means for galaxies with different restframe colors $(\S 4.6)$.

\subsection{Overall performance}

The combined effects of mismatch between true and template SFH, attenuation by dust, metallicity variations and AGN activity on our ability to characterize the stellar population of a galaxy are summarized in Fig. 6 . Here, we plot the best estimate from SED modeling versus the true ${ }^{1}$ value of the considered stellar population property. We bin the distribution of points for different initial conditions, timesteps and lines-of-sight. Darker intensities represent a higher density in the bin. The solid curve represents the median of the distribution and the dotted curves mark the central $68 \%$ interval.

The youngest ages in Fig. 6(a) correspond to the early stages of simulations with an initial gas fraction of $80 \%$. The stars present at the start of these simulations were assigned young ages and low metallicities (see $§ 2.1$ ). In $\S 4.4$, we describe how modeling sub-solar metallicity populations with solar metallicity templates can lead to age overestimates. Since the lower gas fraction runs start with higher stellar ages and metallicities, they do not show this trend. Fig. 6(b) compares the recovered and true stellar mass, normalized to the final stellar mass of the simulation. At low $M / M_{\text {final }}$ ratios, i.e., at the start of the high gas fraction simulations, the mass estimates agree well with the true values. Here, the reason is that biases in age and extinction average out. On the one hand, the overestimated age leads to an overestimate of the intrinsic mass-to-light ratio $\left(\left[\frac{M}{L_{\text {Int }}}\right]_{\text {estimate }}>\right.$ $\left.\left[\frac{M}{L_{\text {Int }}}\right]_{\text {true }}\right)$. On the other hand, the amount of extinction tends to be underestimated $\left(\left[\frac{L_{\mathrm{Int}}}{L_{\mathrm{Att}}}\right]_{\text {estimate }}<\left[\frac{L_{\mathrm{Int}}}{L_{\mathrm{Att}}}\right]_{\text {true }}\right)$. As a result, the mass estimate for this phase is relatively robust:

$$
\begin{aligned}
M_{\text {estimate }} & =L_{\text {Att }}\left[\frac{L_{\text {Int }}}{L_{\text {Att }}}\right]_{\text {estimate }}\left[\frac{M}{L_{\text {Int }}}\right]_{\text {estimate }} \\
& \approx L_{\text {Att }}\left[\frac{L_{\text {Int }}}{L_{\text {Att }}}\right]_{\text {true }}\left[\frac{M}{L_{\text {Int }}}\right]_{\text {true }} \\
& =M_{\text {true }}
\end{aligned}
$$

where $L_{\text {Int }}$ is the intrinsic and $L_{\mathrm{Att}}$ is the attenuated (i.e., observed) luminosity. The largest systematic mass underestimates occur at intermediate $M / M_{\text {final }}$, during the merger-triggered star-forming phases of the simulation. This can be explained in terms of a mismatch between the true SFH and the SFH of the best-fitting template (see $\S 4.2$ ), in combination with an underestimate of

\footnotetext{
1 Hereafter, we use 'true' when referring to the value of a stellar population property as computed using information on all SPH particles in the simulation. Quantities derived by fitting templates to integrated SEDs are referred to as 'estimates'. While 'true' mass and mass-weighted age can be read directly from the simulation, we note that parameters as 'true' reddening and visual extinction depend on the adopted stellar population synthesis code and details of the radiative transfer, which themselves may have biases.
}

the extinction (see §4.3). Briefly, such a SFH mismatch leads to age underestimates, hence $\frac{M}{L_{\mathrm{In}}}$ underestimates, and, added to an insufficient dust correction, to mass underestimates. Finally, the correspondence is best at $\log \left(M / M_{\text {final }}\right) \sim 0$, where the merger remnants reside.

The reddening (Fig. 6(c)) is overall well reproduced. Only at the highest reddening levels, the agreement deteriorates. The latter correspond to the times when and viewing angles under which the effect of increased extinction toward young stars is maximal, as will be explained in $\S 4.3$. As opposed to the reddening, however, the extinction (Fig. 6(d)) shows large systematic underestimates. Using

$$
R_{V}=\frac{A_{V}}{E(B-V)}=4.05
$$

to translate the selective absorption $E(B-V)$ into a total visual absorption $A_{V}$ results in an underestimate over the whole range of $A_{V}$ values, particularly during the highly obscured phases. An observer is limited by the light that he/she receives. In $\S 4.3$, we discuss in depth how the sum of emitting sources that are each attenuated according to Calzetti et al. (2000) does not follow that same reddening law.

Finally, since the reliability of SFR indicators from Xrays over optical and IR to radio wavelengths has been topic of much recent debate (e.g., Reddy et al. 2006; Papovich et al. 2006; 2007; Daddi et al. 2007a), it is interesting to investigate its recovery by modeling the optical-to- $8 \mu \mathrm{m}$ SEDs. This is presented in Fig. 6(e). At low and intermediate SFRs $\left(S F R_{\text {true }}<100 M_{\odot} /\right.$ yr $)$, we find a satisfying agreement, with a median offset that increases slightly towards higher SFRs. For more than $84 \%$ of the galaxies with $S F R_{\text {true }}>100 M_{\odot} /$ yr, we underestimate the SFR, typically by factor of 3 . These systems often suffer strong dust obscuration, and therefore this bias in SFR directly relates to the large underestimates of extinction at the high $A_{V}$ end in Fig. 6(d).

In order to quantify the performance of the SED modeling in estimating stellar mass-weighted ages, we define $\Delta \log \left(a g e_{w}\right)$ as $\log \left(a g e_{w, \text { recovered }}\right)-\log \left(a g e_{w, \text { true }}\right)$. Similar definitions are used to quantify the offset in mass, reddening and extinction, always indicating an underestimate with a negative value of $\Delta=$ parameter $_{\text {recovered }}-$ parameter $_{\text {true. }}$ Fig. 7 presents the performance of the SED modeling on the full photometry (including dust, metallicity variations, and AGN), expressed by the $\Delta$ values, as a function of time since the merger. We adopt the same binned plot style as in Fig. 6, with darker intensities meaning a higher density in the bin. Open boxes contain less than $1 \%$ of the total number of SEDs at that timestep.

We quantify the performance of the SED modeling separately for galaxies in the 'disk', 'merger', and 'spheroid' regime by computing the median and the central $68 \%$ interval of the distribution of $\Delta$ values for all simulation snapshots (under a range of viewing angles) in that phase. We find $\Delta \log a g e_{w, \text { disk }}=+0.03_{-0.42}^{+0.19}$, $\Delta \log a g e_{w, \text { merger }}=-0.12_{-0.26}^{+0.40}$, and $\Delta \log a g e_{w, \text { spheroid }}=$ $-0.03_{-0.14}^{+0.12}$. The underestimate and scatter is largest for the phases of merger-triggered star formation. These are the statistics for low and high gas fraction runs combined. Considering simulations with an initial gas frac- 

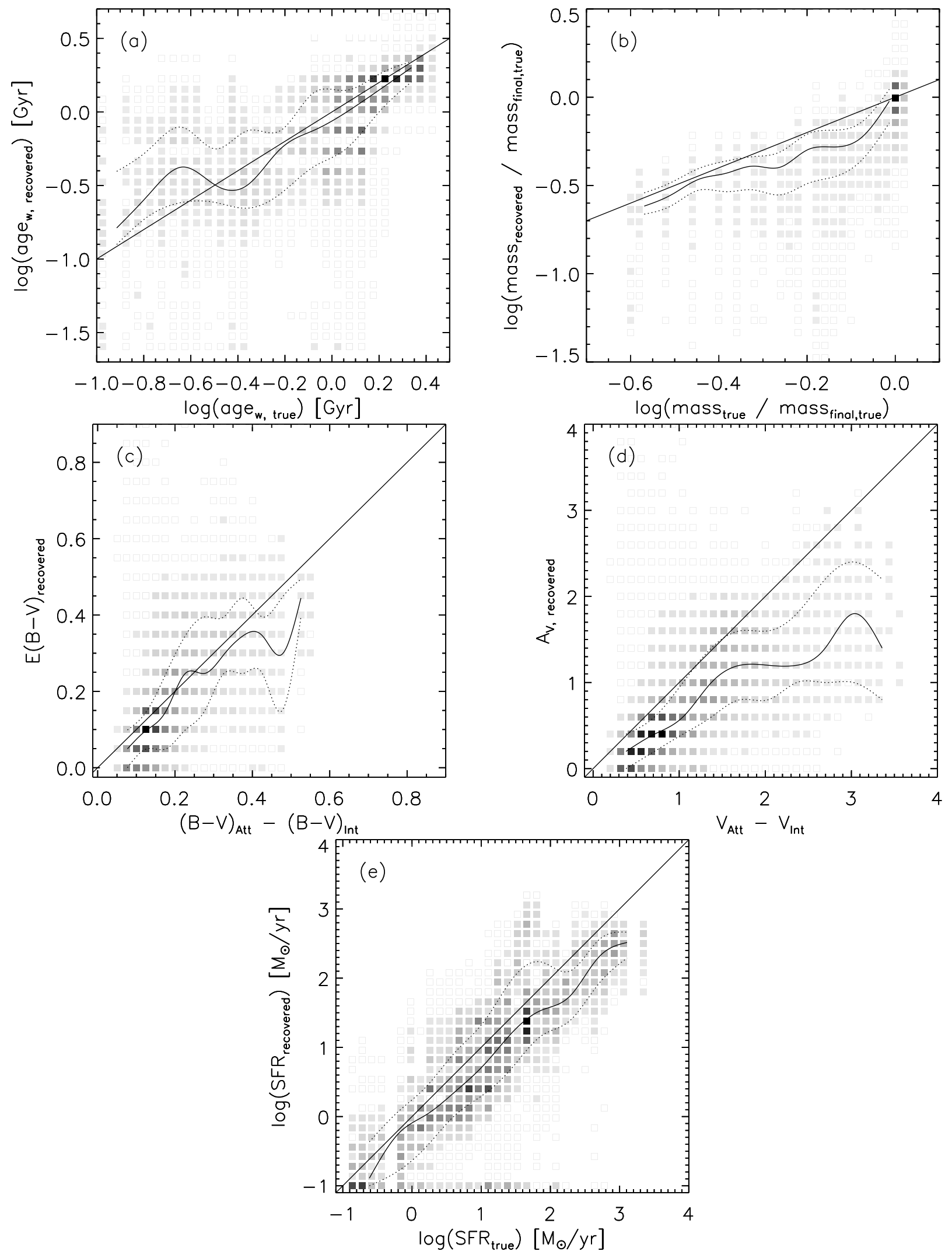

FIG. 6.- Overall performance of the SED modeling. Recovered versus true (a) mass-weighted stellar age, (b) ratio of current to final stellar mass, (c) effective reddening (i.e., attenuated minus intrinsic $B-V$ color), (d) effective visual extinction (i.e., attenuated minus intrinsic V-band magnitude), and (e) star formation rate. The SED modeling was performed on the total (stellar+AGN) attenuated photometry corresponding to simulations with a range of masses, starting with $40 \%$ and $80 \%$ gas fractions, and seen at different timesteps and under different viewing angles. The solid curves indicate the median and dotted curves comprise the central $68 \%$ of the distribution. The total visual extinction $A_{V}$ is the least constrained of the studied parameters. In particular for heavily extincted galaxies the $A_{V}$ is greatly underestimated. 

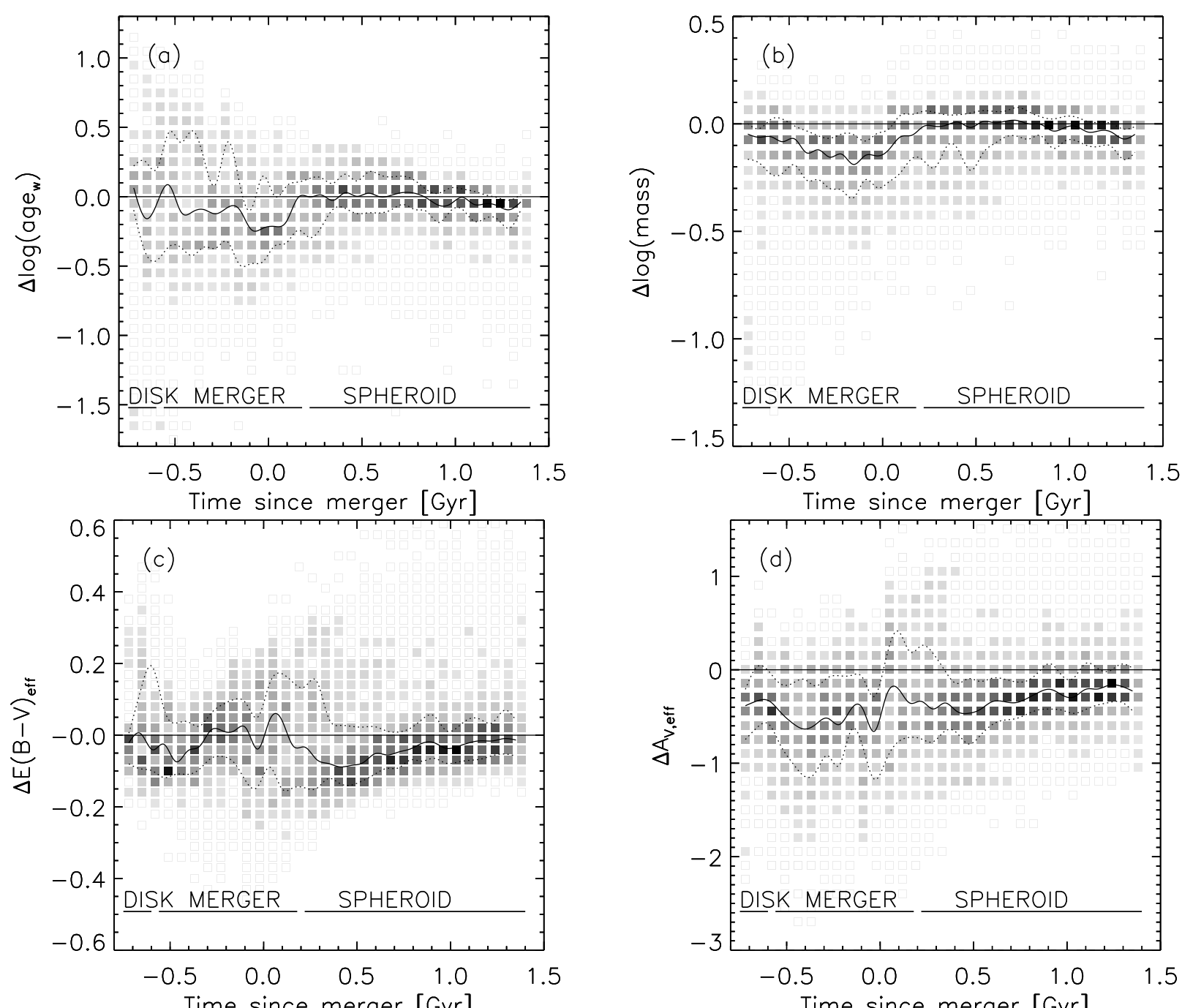

Time since merger [Gyr]

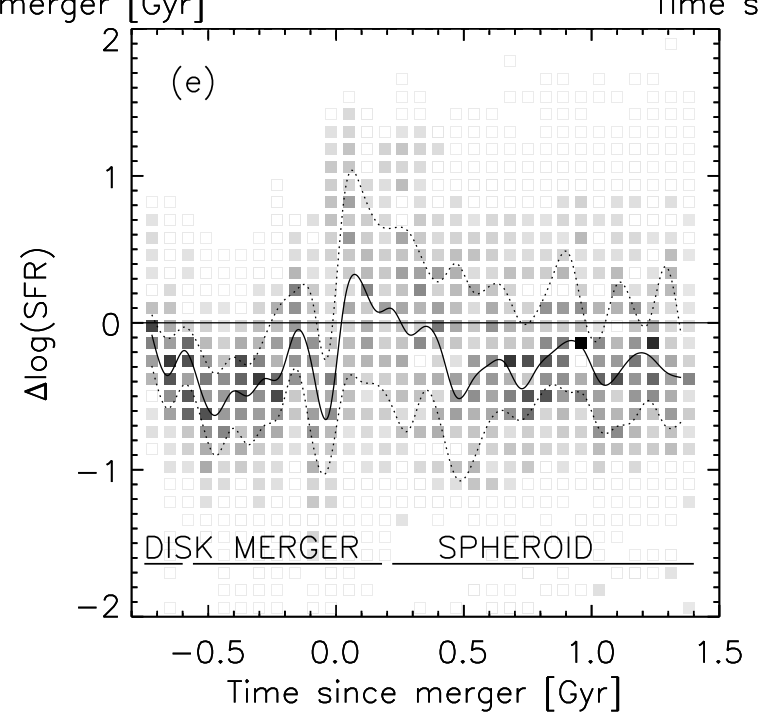

FIG. 7.- Overall performance of the SED modeling. The difference between estimated and true (a) mass-weighted age, (b) stellar mass, (c) effective reddening, (d) effective visual extinction, and (e) star formation rate as a function of time since the merger. The SED modeling was performed on the total (stellar+AGN) attenuated photometry. The solid curves indicate the median and dotted lines comprise the central $68 \%$ of the distribution. The properties of merger remnants are well reproduced. The results for star-forming galaxies, especially for those in the phase of merging, show underestimates in both age, extinction, star formation rate, and mass. 
tion of $40 \%$ separately, the age underestimates during the star-forming phases increase: $\Delta \log a g e_{w, \text { disk } 40 \%}=$ $-0.13_{-0.34}^{+0.25}$ and $\Delta \log a g e_{w, \text { merger }, 40 \%}=-0.30_{-0.11}^{+0.08}$. The $f_{\text {gas }}=80 \%$ runs instead show age overestimates until $\sim 200$ Myr before the merger, mainly due to their lower initial metallicity (see §4.4). During the spheroid phase, ages are recovered similarly well for the low and high gas fraction runs. The recovery of other stellar population properties does not depend strongly on gas fraction unless stated otherwise.

Fig. 7(b) shows the same scenario as described for Fig. $6(\mathrm{~b})$. Namely, mass estimates are robust once the merger remnant phase is reached $\left(\Delta \log M_{\text {spheroid }}=-0.02_{-0.11}^{+0.06}\right)$, but more significant mass underestimates occur for starforming disks $\left(\Delta \log M_{\text {disk }}=-0.06_{-0.14}^{+0.06}\right)$, and especially during phases of merger-enhanced star formation $\left(\Delta \log M_{\text {merger }}=-0.13_{-0.14}^{+0.10}\right)$.

Averaged over all SEDs for a given time with respect to the merger (representing different viewing angles, masses, and gas fractions), our reddening estimates are well behaved: $\Delta E(B-V)_{\text {disk }}=-0.02_{-0.07}^{+0.13}$, $\Delta E(B-V)_{\text {merger }}=-0.02_{-0.08}^{+0.08}$, and $\Delta E(B-V)_{\text {spheroid }}=$ $-0.03_{-0.07}^{+0.11}$. However, as noted already in Fig. 6(d), the bias in estimated visual extinction is often much larger, particularly during the star-forming (disk and merger) phases: $\Delta A_{V, \text { disk }}=-0.35_{-0.34}^{+0.29}, \Delta A_{V \text {, merger }}=$ $-0.54_{-0.46}^{+0.40}$, and $\Delta A_{V \text {,spheroid }}=-0.29_{-0.30}^{+0.32}$. Part of the scatter quoted for $\Delta E(B-V)$ and $\Delta A_{V}$ results from differences between the low and high gas fraction runs. The three key differences, with reference to where their impact is detailed, are the following. First, the high gas fraction runs start out with lower metallicities (§4.4). Second, the low gas fraction runs start with older stars. The larger age range makes the effect of age-dependent extinction more pronounced (§4.3.2). Finally, the high gas fraction runs feed relatively more gas to the $\mathrm{SMBH}$, resulting in a larger AGN contribution to the integrated light during final coalescence $(\S 4.5)$. Since the obscuration during the merger phase tends to be to the newly formed stars mainly, the underestimated extinction leads to an underestimate of the SFR. Consequently, the evolution of $\Delta \log S F R$ with time is as a mirrored version of the star formation history, showing the largest underestimates during first passage and final coalescence. We find $\Delta \log S F R_{\text {disk }}=-0.22_{-0.28}^{+0.23}, \Delta \log S F R_{\text {merger }}=$ $-0.44_{-0.31}^{+0.32}$, and $\Delta \log S F R_{\text {spheroid }}=-0.23_{-0.47}^{+0.62}$. Note that the offset for the spheroid phase merely means that we are unable to distinguish low (a few $0.1 \mathrm{M}_{\odot} / \mathrm{yr}$ ) from very low $\left(\sim 0.1 \mathrm{M}_{\odot} / \mathrm{yr}\right)$ star-forming systems. We are able to robustly classify them as quiescent.

In the above statistics, each $\Delta$ value corresponding to a particular snapshot was given equal weight. Alternatively, we can quantify the performance of our SED modeling in a global sense by summing the SFRs of all snapshots and comparing this to the sum of all recovered SFRs. Such an approach gives more weight to phases with higher SFR, as is also the case for observational studies of the cosmic SFR density. Considering the entire timespan for all simulations, we find that our SED modeling underpredicts the total mass of stars being formed per unit time by a factor 1.8. A similar exercise shows that the total assembled stellar mass is underes-

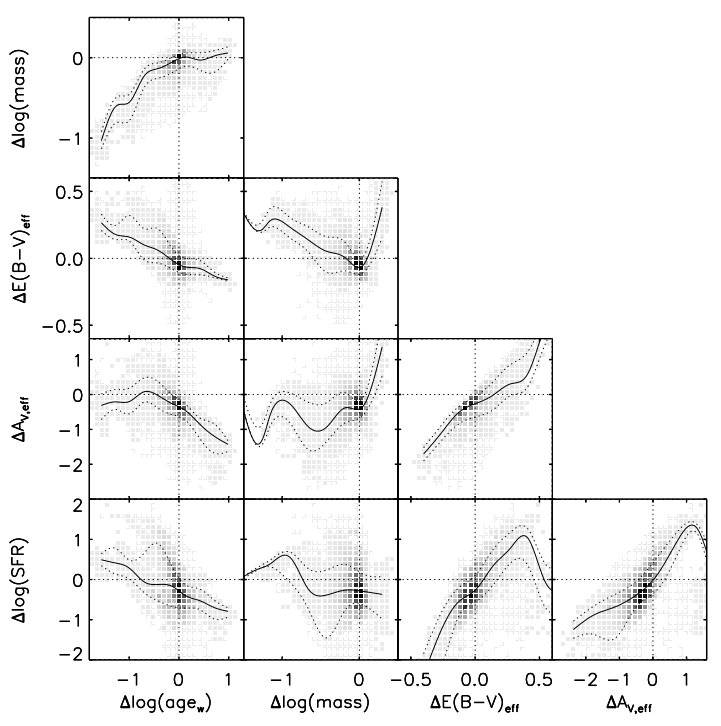

FIG. 8.- Correlations between biases in the estimated age, mass, reddening, extinction, and SFR obtained from broad-band SED modeling. Style as in Fig. 7. Positive values of $\Delta$ mean overestimates, negative values refer to underestimates with respect to the true age, mass, $E(B-V)_{\text {eff }}, A_{V \text {,eff }}$, and SFR in the simulations. Strong correlations exist between $\Delta$ values for different stellar population properties. E.g., over/underestimates in reddening $E(B-V)_{\text {eff }}$ lead to offsets of the same sign in extinction $A_{V \text {,eff }}$ and SFR.

timated by only $14 \%$. When focussing on the merger regime alone, the underestimates increase to a factor 2 for the summed SFR and 30\% for the summed stellar mass.

We present an overview of correlations between the $\Delta$ values of all considered properties in Fig. 8. It is clear that biases in estimates of stellar population properties are often strongly correlated. For example, when the age is severely underestimated, the same will be true for stellar mass. Likewise, a strong positive correlation exists between biases in the estimated reddening, extinction, and star formation rate. In the following sections, we shed more light on how the estimates of age, mass, reddening, extinction, and SFR are connected.

In the light of the upcoming Spitzer warm mission, we note that the interval containing the central $68 \%$ of $\Delta \log M$ values becomes twice as broad when omitting the IRAC bands. This test demonstrates the value of extending SEDs to longer wavelengths. Similar improvements by including IRAC photometry are found for the other stellar population parameters considered in this paper.

We performed our analysis on synthetic photometry of galaxies placed at redshifts $z=1.5$ to $z=2.9$. We note that the results do not change if you change the redshift of the merger. Furthermore, the trends described in this section are all systematic and cannot be attributed to signal-to-noise effects (e.g. more extincted galaxies at the highest redshifts being fainter and therefore less well recovered). We tested this in two ways: first by omitting the perturbation of the synthetic fluxes, second by applying a conservative cut in the observed $K_{s}$-band magnitude: $K_{s, \text { obs }}<23.6$, corresponding to $S / N_{K_{s}}>10$. In both cases, the same trends described in this Section are still present. 


\subsection{Impact of mismatch between true and template SFH}

The biases in estimates of stellar population properties (§4.1) result from a complex interplay between SFH, dust distribution, metallicity variations, and AGN contribution. We now separate these effects in order to obtain a better understanding of the origin of the biases. In this section, we repeat the SED modeling on the intrinsic stellar photometry (i.e., no dust extinction or AGN contribution), with the metallicity of the stars fixed to solar (i.e., no metallicity variations). Each stellar particle is still treated as a SSP with an age given by the GADGET-2 output (i.e., the SFH is identical to that on which the 'full' photometry in $\S 4.1$ was based), but we extract the photometry from solar metallicity SSP models, even if the stellar particles were recorded to have lower/higher metallicities in the GADGET-2 simulation. Since we use solar metallicity templates to compute the integrated light of the simulations as well as to fit the resulting synthetic SEDs, this procedure removes effects due to mismatch in metallicity and isolates the role of the SFH. This illustrates the consequences of a possible mismatch between the true $\mathrm{SFH}$ in the simulation and the allowed template SFHs in our SED modeling. Our aim is to constrain the properties of the bulk of the stars (e.g., mass-weighted age), whereas our input SEDs are obviously of a light-weighted nature. Since massive $\mathrm{O}$ and $\mathrm{B}$ stars make young stellar populations brighter than older stellar populations, giving them more weight in the integrated SED, the light-weighted stellar age will be younger than the mass-weighted stellar age. This is always the case, but provided we have a template representing the correct $\mathrm{SFH}$, it is possible to account for this effect and still find the correct age of the bulk of the stars. Our three allowed SFHs are an SSP, where all stars formed in a single burst, a model with $S F R \propto e^{-t / \tau}$ with $\tau=300 \mathrm{Myr}$, and a constant star formation history. These are standard choices in analyses of distant galaxies. However, they do not encompass a star formation history where the rate of star formation was lower in the past than it is now, as is the case during first passage and during the actual merger-triggered starburst (see Fig. 1). In general, fitting a template SFH that has $\left[\frac{d S F R}{d t}\right]_{\text {template }}<\left[\frac{d S F R}{d t}\right]_{\text {true }}$, the older population will be lost to some degree under the glare of newly formed stars, leading to an underestimate of the age.

This is illustrated in Fig. 9(a), where the difference between recovered and true mass-weighted stellar age is plotted as a function of time since the merger between the SMBHs. During the first snapshot, when the star formation history matches (by construction) our CSF template, we find no systematic offset and a low scatter, purely resulting from photometric uncertainties. Soon after, we start to underestimate the age, with minima coinciding with the moment of first passage (500 Myr before the actual merger) and that of the actual mergertriggered starburst. It is precisely at these moments that the real SFH deviates most from the allowed template SFHs (during the merger, the $\tau_{300}$ and CSF templates are most frequently preferred by the fitting routine). During the starburst phase itself, the median offset of true massweighted age versus recovered age exceeds 0.6 dex, with a large scatter due to differences in the $\mathrm{SFH}$ for different initial conditions. For example, the ratio of SFR at first
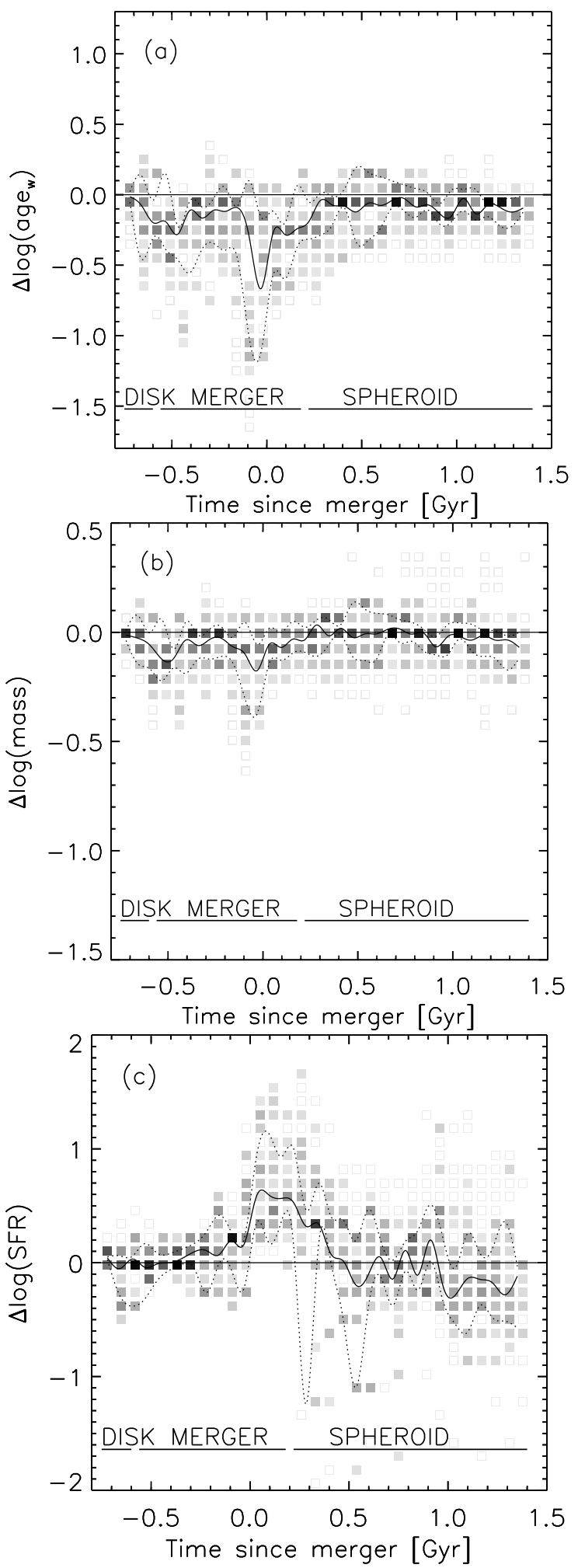

FIG. 9.- Impact of star formation history. The difference between estimated and true (a) mass-weighted age, (b) mass, and (c) SFR as a function of time for all simulations, with the SED modeling performed on the intrinsic (i.e., unattenuated) stellar photometry with all stars set to solar metallicity. Style as in Fig. 7. Deviations from 0 (negative indicating an underestimate) are due to mismatch between the actual star formation history and the histories allowed in our SED modeling (SSP/CSF+dust $/ \tau_{300}+$ dust). Maximal underestimates of age and mass are reached during the merger itself. A secondary minimum is reached during first passage of the progenitors, $\sim 0.4$ Gyr before. The SFR, however, is well recovered during the episodes of merger-enhanced star formation, but not in the first few 100 Myr after. 
passage over SFR during the central starburst increases with gas fraction. After all activity has quieted down, the derived ages lie within 0.1 dex of their true value.

Since one tends to count the young light only, mass will be underestimated as well during first passage and the final nuclear starburst (see Fig. 9(b)). For the same reason, models allowing for a secondary burst of star formation on top of an older stellar population were found to reveal larger total stellar masses, in particular for blue objects (Papovich et al. 2006; Erb et al. 2006b; Wuyts et al. 2007). As for the age measurement, the derived masses for the merger remnants lie within 0.1 dex of their true value.

Since no knowledge about underlying old stellar populations is needed to constrain the ongoing star formation rate, SED modeling of the intrinsic light reproduces the SFR well during the episodes of enhanced star formation (Fig. 9(c)), when age and mass determinations are least reliable. However, the effect of template mismatch between true and best-fit SFH comes into play later. In the simulations, the SFR drops rapidly after reaching its peak during final coalescence. The preferred SFH of the best-fit model most frequently has a slower decrease, over an e-folding time of 300 Myr. By accounting for the bulk of recent star formation, the model consequently overpredicts the ongoing SFR during the first few 100 Myr after the merger, by $\sim 0.6$ dex. Once more time has passed, the SED modeling correctly identifies the remnant as a low SFR galaxy.

We check that the systematic offsets and scatter in Fig. 9 are indeed due to mismatches between the true and template SFHs by performing the following test. For each simulation, we change the distribution of ages of stellar particles to reflect the assumed SFHs that are used in the SED fitting (see $\S 3$ ). We then compute the synthetic photometry in the redshifted passbands as described in $§ 2.2$, still ignoring dust reddening and assuming solar metallicity. Modeling the resulting idealized synthetic SEDs, we recover the mass-weighted age, mass, and SFR essentially without systematic offsets $(\lesssim 1 \%)$, and with little scatter (a fifth of the scatter obtained when the true SFH from the simulations was adopted). The scatter in $\Delta \log M$ decreases by only $40 \%$ when omitting the perturbation of the synthetic fluxes, and for $\Delta \log a g e_{w}$ and $\Delta \log S F R$ the change is even smaller. This indicates that the discrete sampling of the SED by the observed passbands and the discreteness of the age steps allowed in the fit contribute by a similar amount as photometric uncertainties to the very small $\Delta$ values. This test demonstrates that, for a survey as GOODS, mismatch between true and template $\mathrm{SFH}$ is a much larger limitation to constrain stellar population properties of $1.5<z<3$ galaxies than, e.g., photometric uncertainties.

\subsection{Impact of attenuation}

\subsubsection{Non-uniform attenuation uncorrelated to properties of emitters}

As illustrated in $\S 2$, dust attenuation has a large effect on colors, SEDs, and fluxes in all star-forming phases. This is the case for both isolated spiral galaxies (see also Rocha \& Jonsson 2008) and interacting systems (Jonsson et al. 2006). As described in $\S 3$, we use the approach of

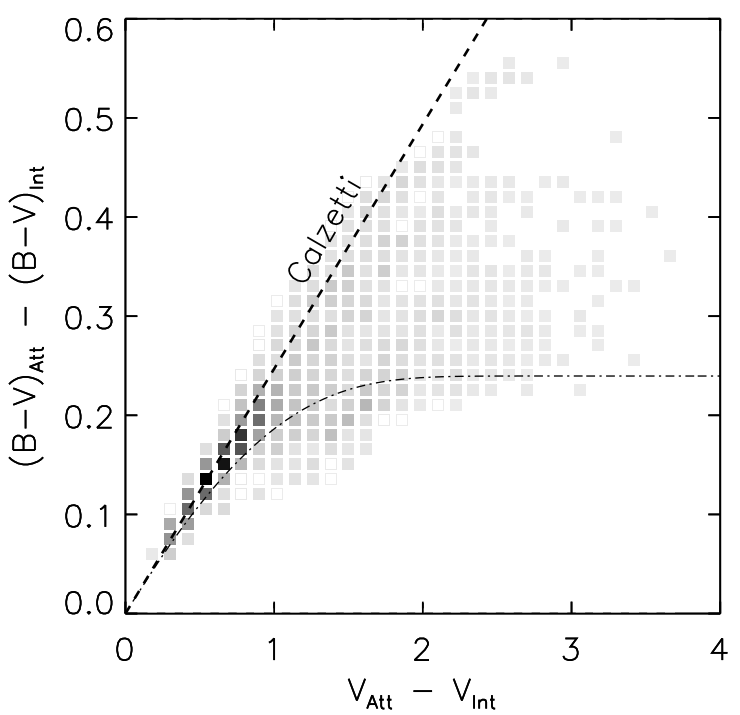

FIG. 10. - The effective reddening (attenuated minus intrinsic $B-V$ color) versus total absorption in the $V$-band for all timesteps, viewing angles and initial conditions. The intensity of the binned distribution indicates the number of simulations in the respective part of the diagram. A ratio of total to selective absorption $R_{V}=4.05$ as by Calzetti et al. (2000) is plotted with the thick dashed line. The dot-dashed curve indicates a series of toy models where the distribution of $A_{V}$ values is uniform between 0 and a maximum value, and all emitting sources are identical. Stellar particles individually have $R_{V}=4.05$, but in the case of a non-uniform dust distribution the sum of all stellar particles has an effective $R_{V}>4.05$.

a uniform foreground screen to account for the attenuation by dust in our SED modeling. Fig. 1(f), illustrating the range of effective visual extinction values (attenuated minus intrinsic $V$-band magnitude) for a random simulation depending on the viewing angle, proves that such a representation is not valid. Here we address the impact that a non-uniform distribution of the dust will have when modeled by a uniform foreground screen.

First, we consider a situation where the optical depth is not the same to all stellar particles, but the variations are uncorrelated with the intrinsic properties of the stellar particles. Such a scenario is by construction the case at the start of the simulation. For each stellar particle individually the ratio of total to selective absorption, $R_{V}=\frac{A_{V}}{E(B-V)}=4.05$, was taken from Calzetti et al. (2000). Since less extincted regions are also less reddened and have a larger weight in the integrated SED, the effective extinction $A_{V \text {,eff }} \equiv V_{\text {Att }}-V_{\text {Int }}$ and effective reddening $E(B-V)_{\text {eff }} \equiv(B-V)_{\text {Att }}-(B-V)_{\text {Int }}$ of the galaxy as a whole will not be related by the same factor 4.05 as for the individual particles. Instead, the overall reddening for a given $A_{V}$ will be smaller than predicted by Calzetti (i.e., the extinction is grayer). This is illustrated in Fig. 10 where the dashed line represents the $A_{V}=4.05 \times E(B-V)$ scaling by Calzetti et al. (2000) and the dot-dashed curve represents a series of toy models for different $A_{V \text {, max }}$ with a uniform distribution of $A_{V}$ values between 0 and $A_{V, \max }$ to stellar particles that all emit at identical intrinsic luminosities. The effective visual extinction for such a model consisting of $\mathrm{N}$ stellar particles emitting with identical luminosities $L_{i}$ is calculated as follows: 


$$
\begin{aligned}
A_{V, \mathrm{eff}} & =V_{\text {Att }}-V_{\text {Int }} \\
& =-2.5 \log \left[\frac{\sum_{i=1}^{N} L_{i} 10^{-0.4 A_{V, i}}}{\sum_{i=1}^{N} L_{i}}\right] \\
& =-2.5 \log \left[\frac{\int_{0}^{A_{V, \max }} 10^{-0.4 A_{V}} d A_{V}}{A_{V, \max }} d A_{V}\right. \\
& =-2.5 \log \left[\frac{10^{-0.4 A_{V, \max }}-1}{-0.4 A_{V, \max } \ln (10)}\right]
\end{aligned}
$$

With $A_{B \text {,eff }}$ calculated in the same way as $A_{V \text {,eff }}$ in Eq. 11 , and using the relation between $A_{B}$ and $A_{V}$ to individual stellar particles from Eq. 7 , the toy model gives an effective reddening of:

$$
E(B-V)_{\mathrm{eff}}=2.5 \log \left[\frac{\left(1+\frac{1}{4.05}\right)\left[10^{-0.4 A_{V, \max }}-1\right]}{10^{-0.4\left(1+\frac{1}{4.05}\right) A_{V, \max }}-1}\right]
$$

It is clear that the relation between total and selective absorption for the series of toy models always lies below the Calzetti relation, and more so for the toy models with the largest $A_{V \text {,eff }}$ (i.e., largest $A_{V \text {, max }}$ ). The reason is that stellar particles with little extinction are relatively dominant in the integrated observed SED. The binned distribution in Fig. 10, presenting total versus selective absorption for all considered simulation snapshots and viewing angles, is roughly encompassed by the Calzetti relation (Eq. 7) on the one hand and our series of toy models (Eq. 11 and Eq. 12) on the other hand.

Since the Calzetti et al. (2000) attenuation law was derived empirically for galaxies as a whole, it is arguably not the appropriate law to apply to the individual stellar particles, i.e., the smallest stellar populations that our simulation can resolve, typically $10^{5}-10^{6} M_{\odot}$. We investigated the changes in photometry when adopting a $\mathrm{MW}$ and SMC-like reddening curve by Pei (1992), which were derived in a more bottom-up fashion from the physics of interstellar dust grains. Again, we scaled the optical depth with the metallicity along the line of sight. For the SMC reddening curve, the resulting colors become redder by up to $0.05,0.1$, and $0.2 \mathrm{mag}$ in rest-frame $B-V$, $U-V$, and $V-J$ respectively. The MW-like attenuation law is also less gray than Calzetti, thus producing slightly redder colors, though less so than for the SMC law. The effective extinction curve, expressed as $\frac{A_{\lambda}}{E(B-V)}$ as a function of wavelength, of snapshots and viewing angles with large optical depths $\left(A_{V \text {,eff }}>1\right)$ is presented for different input attenuation laws in Fig. 11. In each case, we find the typical effective attenuation law to lie above (i.e., show less reddening for a given extinction than) the Calzetti et al. (2000) law. Even in the Kband, such an offset is still notable, and of similar size as would be expected for our toy model with $A_{V \text {, eff }}=2$ (corresponding to $A_{V \text {, } \max }=7$, i.e., many particles are heavily extincted).
Not only does non-uniform extinction change the reddening $\left(\frac{d A_{\lambda}}{d \lambda}\right)$ at a given $A_{V}$, it also affects the dependence of the reddening on wavelength $\left(\frac{d^{2} A_{\lambda}}{d \lambda^{2}}\right)$. For extinction that is uncorrelated to the properties of the emitting sources, this gives the effective dust vector in the $U-V$ versus $V-J$ color-color diagram a shallower slope. I.e., for a given reddening in $V-J$, the reddening in $U-V$ is smaller than predicted by the Calzetti et al. (2000) law. For example, for the simple toy models described in Eq. 11 and Eq. 12, the slope $\frac{A_{U, \text { eff }}-A_{V, \text { eff }}}{A_{V, \text { eff }}-A_{J, \text { eff }}}$ of the effective reddening vector decreases asymptotically with increasing $A_{V \text {,max }}$ to half the slope of the Calzetti reddening vector. The consequence of a different $\frac{d^{2} A_{\lambda}}{d \lambda^{2}}$ than Calzetti is clarified in Fig. 12. The solid curve represents the evolutionary track of a stellar population following a CSF history. The track starts $50 \mathrm{Myr}$ after the onset of star formation and ends 2 Gyr later. Suppose different parts of a galaxy all contain a 1 Gyr old CSF population whose intrinsic location in color-color space is marked by the open gray box. A distribution of dust as described by the above mentioned toy model will redden the galaxy along the dotted line. Interpreting the observed colors (filled circle) as a CSF population attenuated by a foreground screen according to the Calzetti et al. (2000) law, will lead to a best-fit age (star symbol) that is too young and reddening that is too large.

Since in our simulations the ages of the stellar particles (that are each treated as SSPs) present at the start of the simulation were drawn randomly from a uniform distribution, the system has a CSF history in the earliest snapshots without a correlation between the optical depth and intrinsic luminosities and colors of the stellar particles. Therefore, it comes as no surprise that, when looking at the attenuated stellar photometry in Fig. 13 (for now all stars still set to solar metallicity), the central $68 \%$ interval in $\Delta \log a g e_{w}$ reaches to more negative values (to -0.5 dex) during the earliest phases than was the case for the unattenuated photometry (Fig. 9). The estimated reddening is slightly larger than the true value, but nevertheless the use of Eq. 7 still causes an underestimated $A_{V}$, as can be understood from Fig. 10. The systematic underestimate in age and $A_{V}$ combined cause the evaluation of the stellar mass during the first snapshots, when template mismatch due to the SFH is still negligible, to be too small by $\sim 0.12$ dex.

After a few 100 Myr after the beginning of the simulation however, Fig. 13 reveals an improved recovery of the mass-weighted stellar age compared to that obtained by SED modeling of the intrinsic light (Fig. 9). Clearly, the assumption of a non-uniform dust distribution that is uncorrelated with the intrinsic properties of the emitting sources breaks down.

\subsubsection{Preferential extinction toward young stars}

Fig. 14 demonstrates the occurence of preferential extinction toward young star forming regions in one of our simulations. The three panels indicate the binned distribution of the metallicity-scaled hydrogen column density measured along various lines-of-sight versus the age of the stellar particle to which the column density was computed for the 3 snapshots marked in Fig. 1(a). The vertical arrow indicates the start of the simulation. All 


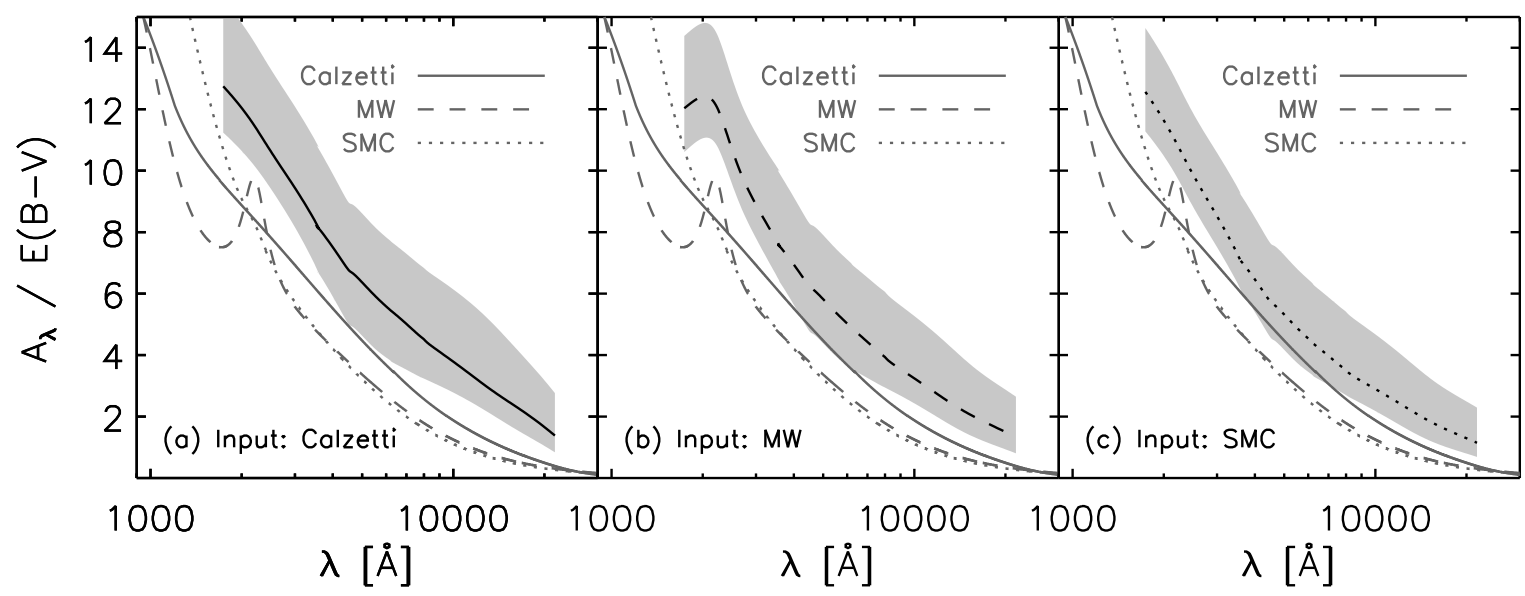

FIG. 11.- Effective extinction curves of simulated galaxies with $A_{V \text {,eff }}>1$ for different input attenuation laws: (a) the Calzetti et al. (2000) law, MW-like reddening from Pei (1992), and (c) SMC-like reddening from Pei (1992). The black curve indicates the median over all snapshots and viewing angles with $A_{V \text {,eff }}>1$. The light gray polygon indicates the central $68 \%$ interval. The Calzetti, MW, and SMC attenuation laws are plotted in gray. In all cases, the effective extinction of simulated galaxies with large $A_{V \text {, eff }}$ is grayer than the Calzetti et al. (2000) law that is used in standard SED modeling. The offset is smallest when each stellar particle is attenuated according to the SMC-like law.

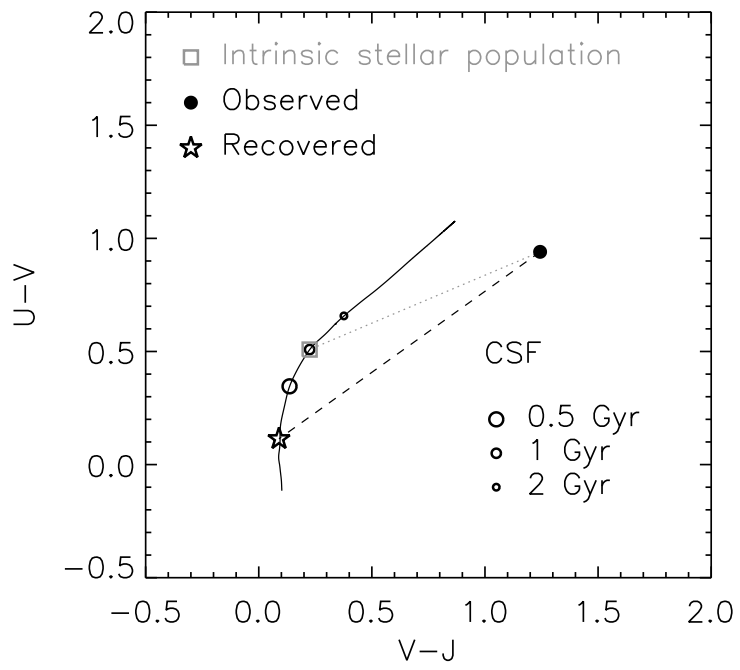

Fig. 12.- Rest-frame $U-V$ versus $V-J$ color-color diagram illustrating the effect of a non-uniform distribution of $A_{V}$ values that is uncorrelated with the intrinsic properties of the emitting sources. The black curve indicates a CSF population with age between 50 Myr and 2 Gyr. Suppose an intrinsic population of age 1 Gyr (box symbol) is reddened by such a dust distribution to the location in color-color space of the filled circle. Under the assumption of a uniform foreground screen of dust, the observed colors will then be traced back along the Calzetti et al. (2000) reddening vector (dashed black line), resulting in an artificially young age (star symbol).

stellar ages older than this value (cut off for illustrational purposes) were set by hand as explained in $\S 2.1$. As we already pointed out in Fig. 1(f), the typical column densities are higher during the merger (panel $b$ ) than before (a) or after (c). Moreover, Fig. 14 shows that the ratio of column densities toward ongoing star formation over column densities toward older populations reaches a maximum during the merger (b). Using sticky particle simulations of dusty starburst mergers, Bekki \& Shioya (2001) found a similar age-dependent extinction, confirming that this is a generic feature of merging systems and not determined by the method used to model dis- sipative processes. Poggianti \& Wu (2000) inferred agedependent extinction during a starburst to explain the nature of so-called $\mathrm{e}(\mathrm{a})$ galaxies: galaxies with $[\mathrm{OII}]$ in emission and strong Balmer absorption lines, frequently associated with merger morphologies.

From a physical perspective, it is expected that during the merging process hydrodynamical and gravitational forces channel gas and dust to the central regions where it triggers a starburst. Once started, supernovae going of on a few $10^{7}$ yr timescale further increase the dust content of the regions where newly formed stars reside. The fact that the distribution of younger (and thus intrinsically bluer) stellar populations does not trace that of the older populations of stars and that it is intimately correlated with the dust distribution leads to an overestimate in age. In Fig. 12, a distribution of $A_{V}$ values that was uncorrelated to the intrinsic properties of the emitters caused an effective reddening along a shallower slope in the $U-V$ versus $V-J$ diagram than for the Calzetti curve. In case of a distribution of $A_{V}$ values that is correlated with the intrinsic colors of the emitters (larger $A_{V}$ to intrinsically bluer stellar populations), the slope of the effective reddening vector in the $U-V$ versus $V-J$ diagram is steeper than for the Calzetti et al. (2000) law. Since an observer will mistakenly model the galaxy with an intrinsically redder template, the reddening by dust $E(B-V)$ will be underestimated. Although a given total absorption corresponds to a stronger reddening in the presence of age-dependent extinction compared to uncorrelated non-uniform extinction, the Calzetti et al. (2000) relation between $E(B-V)$ and $A_{V}$ given by Eq. 7 can still be considered as an upper limit. Therefore, the total absorption will be underestimated. This is illustrated in Fig. 10 where we plotted the binned distribution of true $E(B-V)$ versus true $A_{V}$ for all of our simulation snapshots, viewed under a range of viewing angles. Finally, the derived stellar mass owes its more robust character to the compensating effects of systematic offsets in age and absorption.

The effect of the larger extinction toward young stars will in practice be superposed on the effect of mis- 

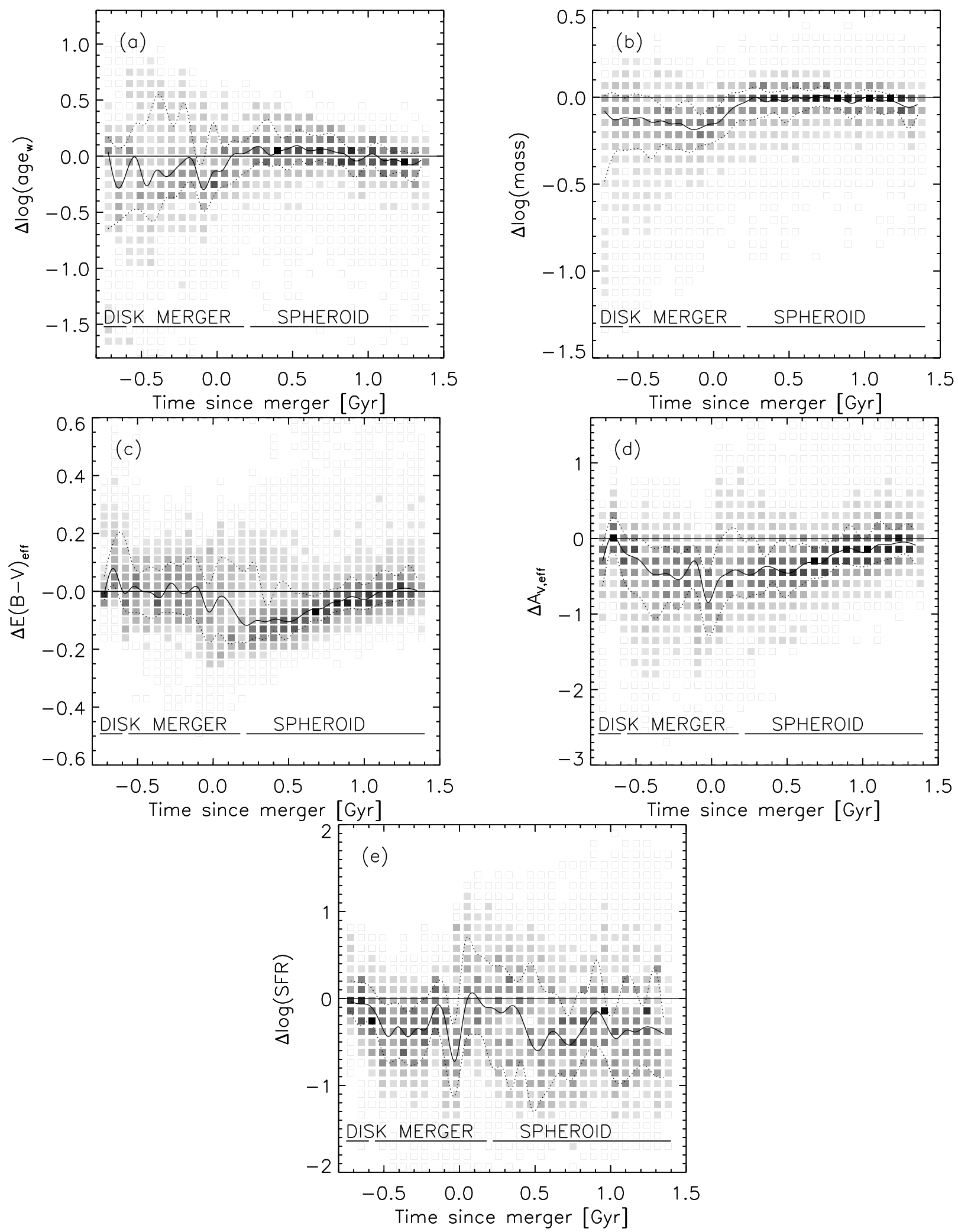

FIG. 13.- Effect of extinction. The difference between estimated and true (a) mass-weighted age, (b) stellar mass, (c) effective reddening, (d) effective visual extinction, and (e) star formation rate as a function of time since the merger. The SED modeling was performed on the attenuated stellar photometry with all stars set to solar metallicity. Style as in Fig. 7 . Ages are still underestimated for the first 0.8 Gyr of the evolution, but to a lesser degree than estimates based on the intrinsic light. Added to the underestimated $A_{V}$, this leads to a characterization of the stellar mass that is too low by $0.1-0.15$ dex. The recovery of SFRs shows minima around first passage ( -0.4 Gyr) and final coalescence (-0.1 to 0 Gyr). 

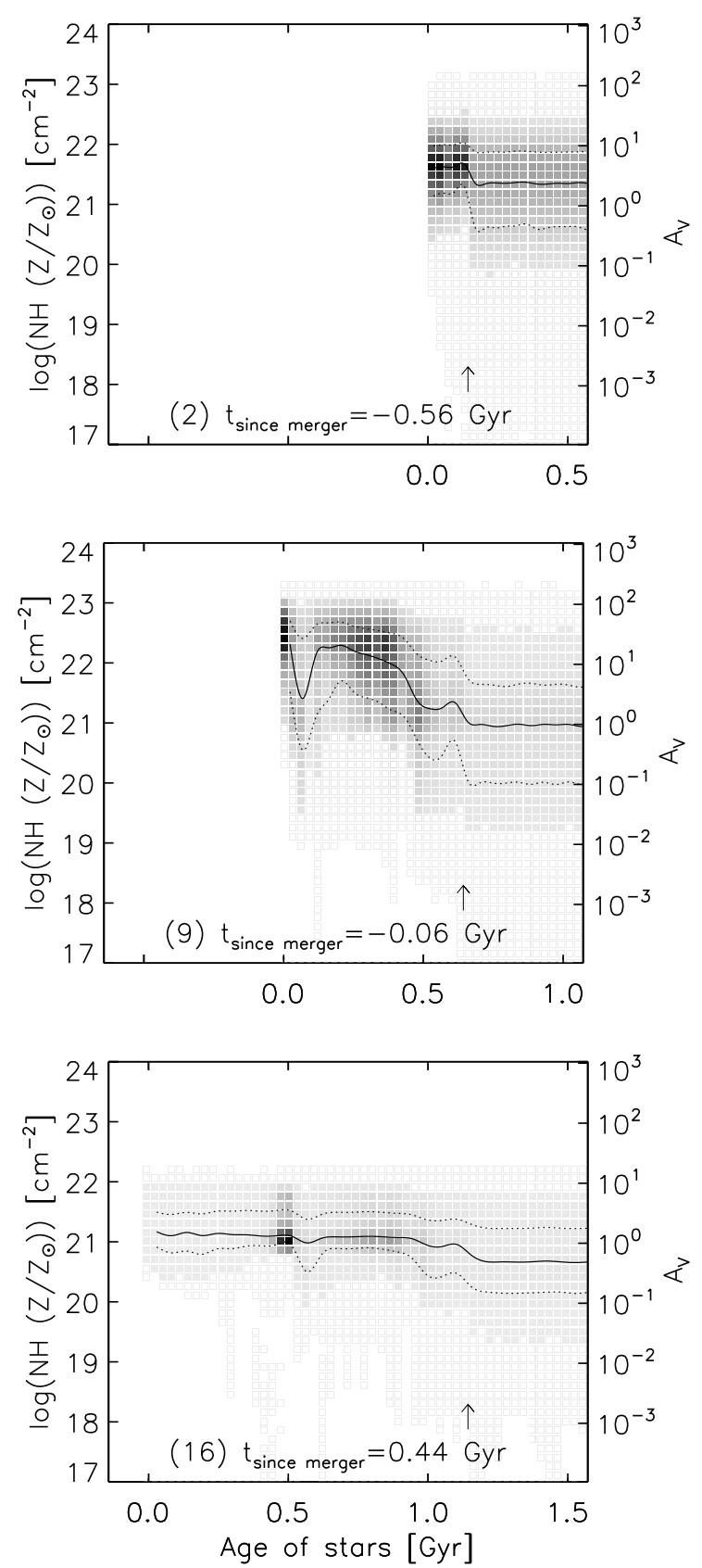

FIG. 14.- Distribution of hydrogen column densities, linearly scaled with the metallicity of the gas along different lines-of-sight to the stellar particles versus the age of the respective stellar particle. The relation between column density and stellar age is plotted for 3 snapshots: before (2), during (9), and after (16) the merger (see Fig. 1). The solid and dotted curves indicate the median and $68 \%$ interval of the distribution respectively. Darker intensity means a larger number of stars is present with that age. All stellar ages rightward of the arrow correspond to initial stars and were set by hand. The optical depth, which is proportional to the metallicityscaled gas density, is larger toward newly formed stars during the merger-triggered starburst. The signature of this age-dependent extinction weakens during more quiescent episodes of star formation.

match between template and true SFH, that prevents us from fully accounting for the difference between lightand mass-weighted stellar age (see §4.2). Fig. 15 illustrates how an increased extinction toward young stars reduces the difference between the light-weighted and

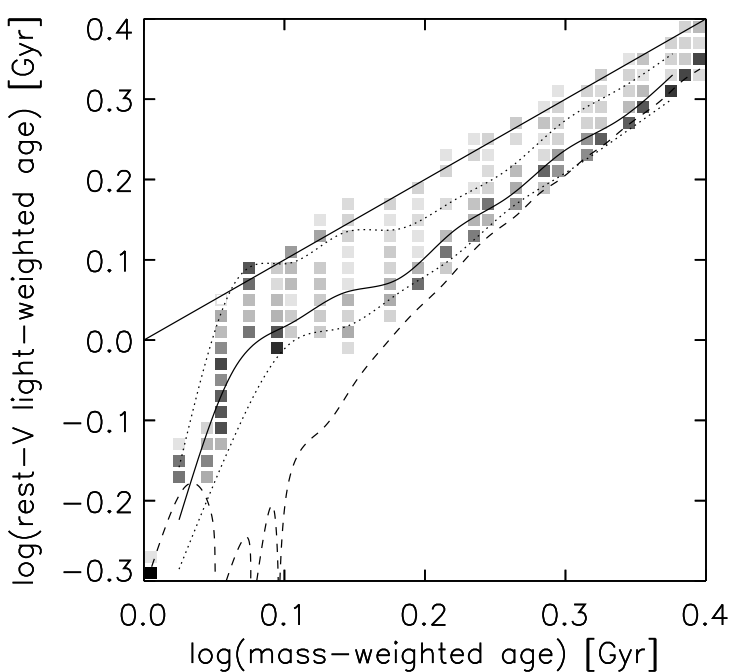

FIG. 15.- Rest-frame attenuated and intrinsic $V$-band lightweighted age versus mass-weighted age for an initially $40 \%$ gas fraction simulation. The boxes mark the mean age weighted with the attenuated $V$-band light. Darker intensities indicate a larger number of viewing angles. The solid and dotted curves mark the median and central $68 \%$ interval respectively. The dashed curve indicates the mean age weighted with the intrinsic $V$-band light (no attenuation). The attenuated light-weighted age is a better approximation of the mass-weighted age than the intrinsic light-weighted age, increasingly so for younger stellar populations. Larger optical depths to young than to old stars are responsible for this effect.

mass-weighted measure of age. We conclude that the SED modeling on galaxies with solar metallicity stars and dust distributed in between still underestimates the age during the merger-enhanced star-forming phases, but adding dust has improved our best guess to an overall median offset of -0.04 dex (compare Fig. 13(a) to Fig. $9(\mathrm{a})$ ). Similar conclusions were drawn by Bell \& de Jong (2001) who examine the reddening and dimming effects of dust and its impact on estimating stellar mass-to-light ratios. At later times, the merger remnant is largely devoid of gas and dust, making accurate determinations of age and mass possible, as described already in $\S 4.2$.

Finally, the evolution of $\Delta \log S F R$ over time (Fig. $13(\mathrm{e}))$ differs significantly from that obtained from modeling the intrinsic light (Fig. 9(c)). With the SED modeling now performed on attenuated photometry, we find underestimates in the SFR of -0.4 dex and -0.7 dex during first passage and final coalescence respectively. The agreement between recovered and true SFR improves during the first few 100 Myr after the starburst. Later, during the spheroid phase, large values of $\Delta \log S F R$ merely reflect our inability to distinguish between low (a few $\left.0.1 M_{\odot} / y r\right)$ and very low $\left(\sim 0.1 M_{\odot} / y r\right)$ SFRs. We therefore focus on the underestimates during the star-forming phases. In the presence of dust, there is a component of the merger-triggered starburst that is completely enshrouded. This allows young stars to be hidden without revealing their presence by an increased reddening of the integrated SED. Since these heavily obscured star-forming regions are not accounted for by the bestfit model, the SFR during these phases is significantly underestimated. 


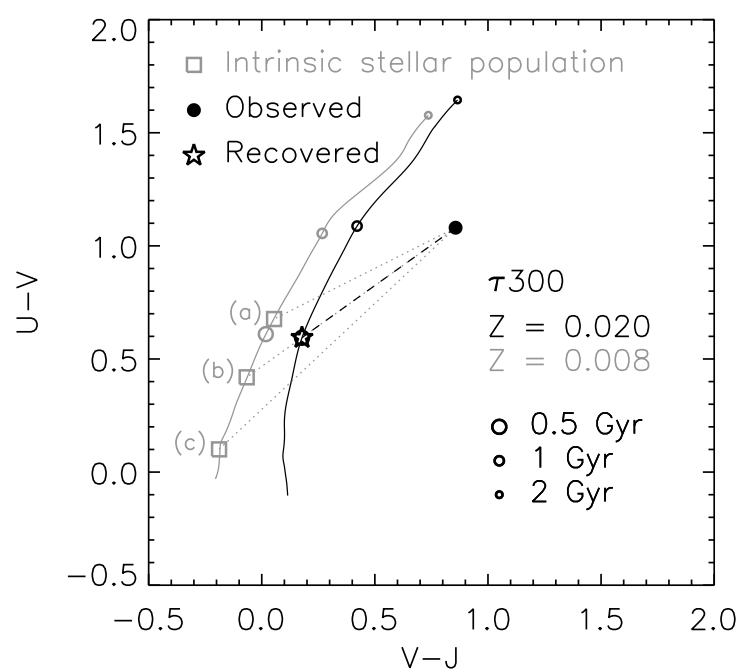

FIG. 16. - Rest-frame $U-V$ versus $V-J$ color-color diagram illustrating the effect of fitting solar metallicity templates to stellar populations of sub-solar metallicity. The black and gray curves represent evolutionary tracks for an exponentially declining star formation history with $e$-folding time of $300 \mathrm{Myr}$ for $\operatorname{solar}(Z=0.02)$ and sub-solar $(Z=0.008)$ metallicity respectively, each starting at 50 Myr. A stellar population with intrinsic colors indicated by the gray boxes will be reddened to the location in color-space marked by the filled circle in the case of (a) non-uniform age-independent extinction, (b) extinction by a uniform foreground screen, and (c) age-dependent extinction. In all three cases, the assumption of solar metallicity and Calzetti attenuation will lead to the conclusion that the stellar population formed its first stars 0.5 Gyr ago. This is an underestimate $(a)$ or overestimate $(b, c)$ respectively. The reddening is always underestimated.

\subsection{Impact of stellar metallicity}

In $\S 4.2$ and $\S 4.3$, we tested our SED modeling on synthetic photometry that was computed assuming a solar metallicity for all emitting sources. In reality, stars with a range of metallicities will be present, reflecting the level of enrichment in the gas at the epoch of their formation. Before we repeat our analysis now setting the stellar metallicities to their appropriate value calculated by the GADGET-2 code, we anticipate the effect using the diagnostic $U-V$ versus $V-J$ color-color diagram in Fig. 16.

The tracks represent exponentially declining SFHs for metallicities of $\mathrm{Z}=0.008$ (gray) and 0.02 (solar, in black). Both evolutionary tracks are drawn from $50 \mathrm{Myr}$ to 2 Gyr after the onset of star formation. The classic agemetallicity degeneracy states that the optical broad-band colors of a young stellar population are nearly indistinguishable from that of an older, more metal-poor population (O'Connell 1986). For the $\tau_{300}$ star formation history drawn here, this effect becomes only notable at later times: 2 Gyr after the onset of star formation the sub-solar metallicity track has the same $U-V$ color as a solar metallicity population that started forming stars 1.8 Gyr ago. On the one hand, the addition of dust will complicate the age-metallicity degeneracy. On the other hand, the addition of rest-frame NIR photometry helps to separate the evolutionary tracks for different metallicities (see Fig. 16). A galaxy whose attenuated light has colors marked by the filled circle may correspond with one of the intrinsic colors indicated by the gray boxes depending on the kind of extinction: (a) for non-uniform age-independent extinction, (b) for a foreground screen of dust, and (c) for age-dependent extinction. In case (a), the assumption of Calzetti attenuation and solar metallicity in our SED modeling leads to a recovered evolutionary stage that is too young, marked with the star symbol on the solar metallicity track. In case (b) and (c), the same recovered evolutionary stage is too old. In all cases, the determination of the reddening will be too low, as will consequently be the case for the $A_{V}$ and the stellar mass, and increasingly so for lower metallicities. Obviously, the effects described will again be superposed on the previously discussed effects of star formation history and dust. It is also noteworthy that taking into account the enrichment by heavy elements reduces the effect of age-dependent extinction. Young stellar populations are still intrinsically bluer than old populations, but to a lesser degree since they have formed at later times from gas that was more enriched.

In our recovery analysis of stellar population properties, we find that at metallicities of a quarter solar and below, the age is overestimated by 0 to 0.5 dex (central $68 \%$ interval of $\left.\Delta \log a g e_{w}\right)$. However, the underestimate in reddening and therefore extinction for these low-metallicity galaxies is such that the mass estimate (which is dependent on both age and $A_{V}$ ) stays within \pm 0.1 dex of its true value for $68 \%$ of the cases. We find no dependence of $\Delta \log S F R$ on metallicity.

\subsection{Impact of $A G N$ contribution}

Since the merger simulations described in this paper take into account the role of supermassive black holes on its environment (see e.g. Di Matteo et al. 2005; Springel et al. 2005b), we now include its contribution to the integrated galaxy SED. We note that only during a timespan of the order of a Salpeter time, a few $10^{7}$ to $10^{8}$ year, the AGN emission amounts to a significant fraction of the stellar emission. Admittedly, the peak of AGN activity can be missed by the time sampling of our snapshots. Nevertheless, the current dataset provides a useful insight on its impact on the SED modeling.

We illustrate the typical behavior in Fig. 17 showing the photometry computed at the time of merging when the accretion onto the supermassive black hole is maximal. Here, the solid black curve represents the light received by an observer. We break down the attenuated SED in a stellar (dotted curve) and AGN contribution (dashed curve). Finally, the best-fit model (in this case an exponentially declining star formation history that started 0.8 Gyr ago) is plotted in gray. Although resulting in a low $\chi_{\text {reduced }}^{2} \sim 1$, the SED modeling is mislead by a degeneracy between the stellar+AGN light and the stellar light of a younger population obscured by large columns of dust. The addition of AGN light, when exceeding $10 \%$ of the total emission, adds another -0.1 to 0.15 dex to $\Delta \log a g e_{w},+0.05$ to +0.1 mag to $\Delta E(B-V)$, and +0.3 to +0.5 mag to $\Delta A_{V}$. Since all observed light is treated as having a stellar origin, the star formation rates during the phase of and under viewing angles with significant AGN contribution to the observed SED are overestimated by an order of magnitude. These cases typically show a larger $\chi_{\text {reduced }}^{2}\left(70 \%\right.$ have $\left.\chi_{\text {reduced }}^{2}>5\right)$. As expected, simulations with an initial gas fraction of $80 \%$ have relatively more stars formed during final coalescence and more gas fed to the supermassive black hole 


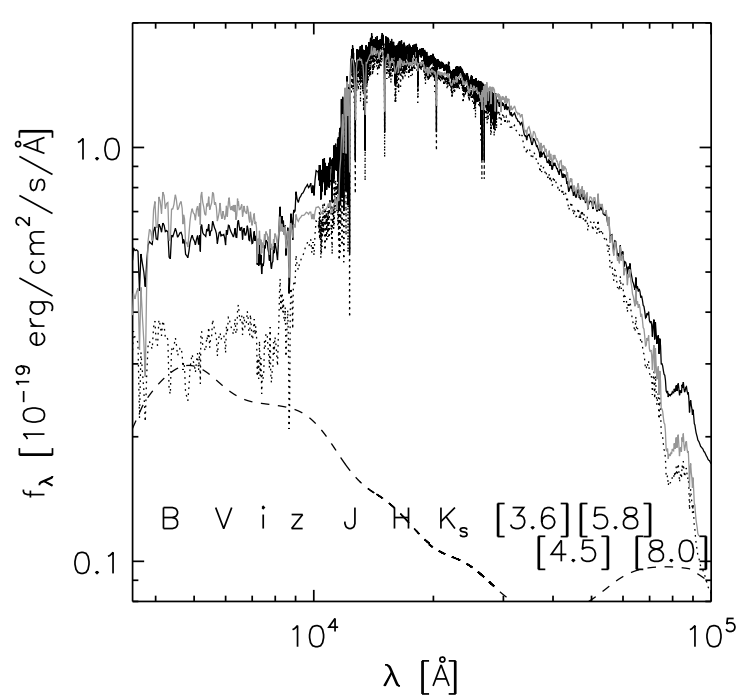

FIG. 17.- Attenuated spectrum of a simulated merger placed at $z=2.1$ during the peak of AGN activity. The total attenuated light (black solid curve) is decomposed into a contribution from stars (dotted curve) and AGN (dashed line). An observer who samples the total attenuated light with an identical set of broad-band filters as available for GOODS-CDFS and models the SED using stellar population synthesis only, will find as best-fitting model the spectrum in gray. Its age is too young by $\Delta \log a g e_{w}=-0.4$. The reddening $E(B-V)$ and absorption $A_{V}$ are overestimated by 0.1 and $0.4 \mathrm{mag}$ respectively. The opposite sign of offsets in age and $A_{V}$ leads to a mass recovery that is only 0.05 dex below its true value.

than lower gas fraction runs. Hence the bias due to a contribution of AGN light is more prominent for simulations with a higher gas fraction.

\subsection{Lessons for SED modeling}

How can the modeling of real high-redshift galaxies benefit from our analysis of merger simulations? In order to answer this question, we need to translate the mechanisms described above in terms of the physical properties of stars and dust into their combined effect as a function of observables.

An observable that correlates crudely with time since the merger, the parameter as a function of which we quantified the quality of SED modeling, is specific star formation rate $(s S F R=S F R / M)$. Generally, merger remnants are characterized by a low $S S F R \lesssim 10^{-9.5}$ $\mathrm{yr}^{-1}$, whereas the sSFR is large $\left(S S F R \gtrsim 10^{8.5} \mathrm{yr}^{-1}\right)$ during the merger-triggered starburst. However, very gas-rich isolated galaxies (the 'disk' phase of simulations with $f_{\text {gas,init }}=80 \%$ ) may reach similarly high sSFRs.

Since the results of our SED modeling showed no trend with redshift for which the synthetic photometry was computed, we can also describe the quality of recovering stellar population properties in terms of rest-frame colors. Although not true observables, their computation as described by Rudnick et al. (2003) suffers from only a minor template dependence compared to parameters such as mass, age, dust content, and SFR. In Fig. 18 , we present the performance of our SED modeling as a function of location in the rest-frame $U-V$ versus $V-J$ color-color diagram. Simulations with initial gas fractions of $40 \%$ and $80 \%$ are shown separately. The SED modeling was based on the full (stellar+AGN) photometry in both cases. Downward triangles indicate a me- dian value of $\Delta=$ parameter $_{\text {recovered }}-$ parameter $_{\text {true }}$ that is negative for simulations with the respective colors. Upward triangles represent an overprediction of the true value. Lighter symbols are used for a better correspondence between the true and modeled parameter value. The different initial stellar ages and lower metallicities for $f_{\text {gas }}=80 \%$ runs explain why they extend to bluer $U-V$ colors. At blue optical colors $(U-V<1)$, the attenuation is seriously underestimated. An overestimate of the age by a similar order of magnitude (at $U-V<0.65$ mostly due to the lower metallicity and at $0.65<U-V<1$ due to age-dependent extinction) leads to a relatively robust estimate of the mass.

At red optical colors $(U-V>1)$, sources with relatively blue $V-J$ colors are generally better modeled than those with the reddest $V-J$ colors in our sample. In the $1<U-V<1.8$ color regime, the effects of dust attenuation play an important role. Here we find objects that are heavily extincted during the merger-triggered starburst, but also disk galaxies seen edge-on during the earliest phases of the simulation. All sources with $V-J>1.5$ in our sample belong the latter category. Since we did not impose an age gradient for the initial stars, the dust distribution in thoses cases is non-uniform but uncorrelated with intrinsic color of the stellar particles. This explains why they have the strongest overestimate in $E(B-V)$ (see §4.4). The upper part of the color-color distribution $(U-V>1.8)$ is where each galaxy ends up by aging without further inflow of gas. In such a system, the $A_{V}$ values are modest to low and the lack of a template exactly matching the SFH is less problematic as the epoch of major star formation lies further back in time.

\section{RESULTS FROM SED MODELING WITH FREE REDSHIFT}

In practice, complete spectroscopic surveys of masslimited samples of high-redshift galaxies are rare. Consequently, we often are not able to fix the redshift in the SED modeling procedure to its exact true value. Over the past few years, several codes have improved on estimating the redshift based on the broad-band SED, by experimenting with different template sets and fitting algorithms. In this section, we will use the new photometric redshift code EAZY (Brammer et al. in preparation) to establish the quality of photometric redshift $\left(z_{\text {phot }}\right)$ estimates derived from our synthetic photometry and analyze the impact of $z_{\text {phot }}$ uncertainties on the derived stellar population properties.

\subsection{The photometric redshift code EAZY}

Here, we summarize the main characteristics of the EAZY photometric redshift code. A full description of the algorithm and template sets will be presented by Brammer et al. (in preparation). We test our ability to recover the redshifts using a template set based on a set of $\sim 3000$ PÉGASE 2.0 (Fioc \& Rocca-Volmerange 1997) templates. The large number of templates was reduced to 5 principal components necessary to span the colors of galaxies in the semi-analytic model by De Lucia \& Blaizot (2007). In addition to these 5 templates, a dusty template with an age of $50 \mathrm{Myr}$ and $A_{V}=2.75$ accounts for the existence of dustier (and thus redder) galaxies than present in the semi-analytic model.

The code fits a non-negative superposition of templates 


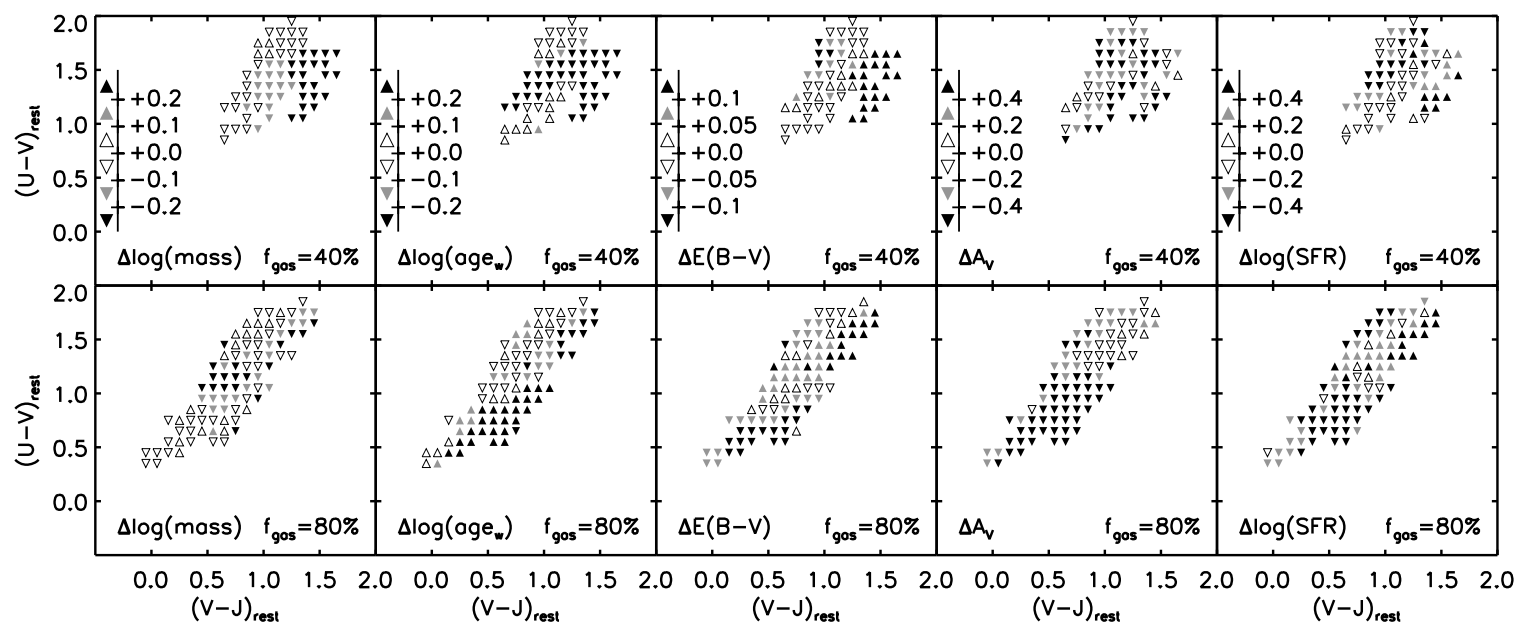

Fig. 18. - Median quality of recovered stellar population properties for simulations with a gas fraction of $40 \%$ and $80 \%$ as a function of rest-frame optical and optical-to-NIR color. Downward triangles indicate underestimates with respect to the true value. Upward triangles mark overpredictions. Galaxies in regions with white triangles are characterized most accurately. Simulations with $f_{\text {gas }}=80 \%$ reach bluer colors in $(U-V)_{\text {rest }}$ than those with $f_{\text {gas }}=40 \%$ since their initial stars were set to younger ages (see $\left.\S 2.1\right)$. At red optical colors $\left[(U-V)_{\text {rest }}>1\right]$, galaxies with relatively blue $(V-J)_{\text {rest }}$ colors are better recovered than those at the red $(V-J)_{\text {rest }}$ end. The former are older, less obscured systems, while the latter have a young and dusty nature. At blue optical colors $\left[(U-V)_{\text {rest }}<1\right]$, large systematics in the determination of age and $A_{V}$ occur. Their opposite signs cancel out in the derivation of stellar mass.

to the $B$-to- $8 \mu \mathrm{m}$ photometry, using a template error function that effectively downweights the rest-frame UV and NIR portion of the templates in the fit. The value of the redshift marginalized over the total redshift probability distribution is adopted as best $z_{\text {phot }}$ estimate.

EAZY allows for the use of a magnitude prior function, constructed from observed or simulated number counts as a function of apparent magnitude and redshift. However, since we shift each simulation over the entire redshift range $1.5 \leq z \leq 2.9$, our methodology does not allow to test this feature.

\subsection{Recovering redshifts and stellar population properties from broad-band photometry}

\subsubsection{Recovering redshifts}

In Fig. 19, we compare the photometric redshifts obtained with EAZY with the input redshifts for which the synthetic broad-band SEDs were computed. The measure commonly used to quantify the photometric redshift quality is $\Delta z /(1+z)$. Its normalized median absolute deviation is 0.031 . We find no dependence of $\Delta z /(1+z)$ on redshift, time since (or before) the merger, or massweighted age. A slight trend with $A_{V}$ was found in the sense that the median $\Delta z /(1+z)$, which varies between -0.01 and 0.01 for $0<A_{V}<2.5$, increases to a few 0.01 for simulated galaxies with $A_{V}>2.5$. However, this systematic offset is still smaller than the scatter in $\Delta z /(1+z)$ for these highly obscured galaxies. The performance of EAZY is very good and competitive with that of other codes presented in the literature. We note that the high $z_{\text {phot }}$ quality owes greatly to the dense sampling of the rest-frame UV-to-NIR SED by our synthetic photometry (filter set and depths were adopted from the GOODS-South survey). Leaving out 1 of the 11 filters, especially when located near a spectral break at the considered redshift, may boost the uncertainty in $z_{\text {phot }}$. E.g., running EAZY on the synthetic SEDs without $H$-band results in a scatter over the $1.5 \leq z \leq 2.9$ range that is $35 \%$ larger: $\sigma_{\mathrm{NMAD}}(\Delta z /(1+z))=0.042$. At $z \sim 2.7$,

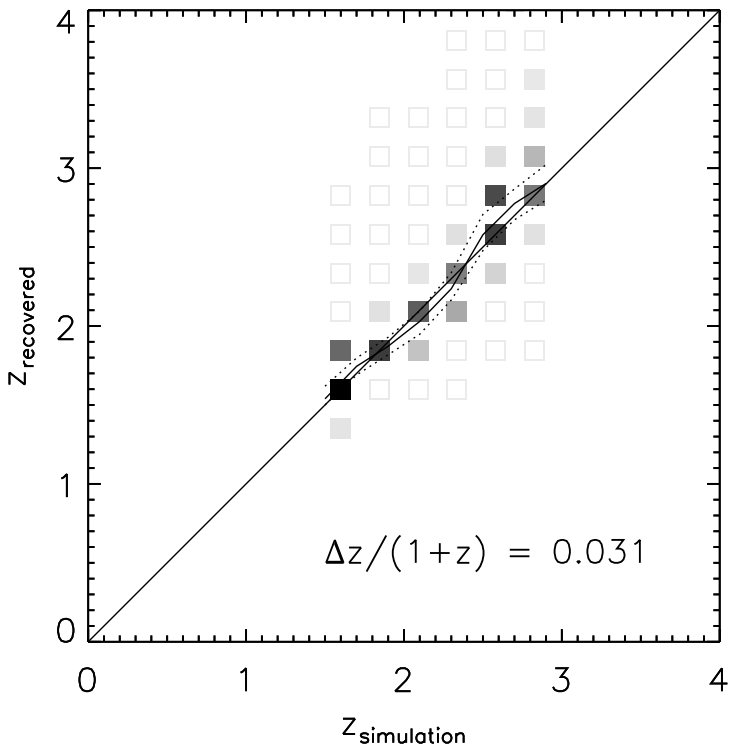

FIG. 19.- Comparison of photometric redshifts by EAZY versus true redshift. The correspondence is good $\left(\sigma_{\mathrm{NMAD}}(\Delta z /(1+z))=\right.$ $0.031)$, without significant systematic offsets.

where the Balmer $/ 4000 \AA$ break has shifted in between $J$ and $H$, we find a median offset and scatter in $\Delta z /(1+z)$ of $0.050\left(z_{\text {phot }}\right.$ being larger than $\left.z_{\text {spec }}\right)$ and 0.071 respectively when omitting the H-band.

This exercise offers a valuable complementary test to the empirical comparison with spectroscopic samples of high-redshift galaxies. The latter are direct measurements and therefore insensitive to our knowledge of stellar tracks and population synthesis. On the other hand, spectroscopic samples of high-redshift galaxies often suffer from selection biases, especially against galaxies lacking emission lines.

\subsubsection{Impact of $z_{\text {phot }}$ uncertainties}


Having quantified the quality of photometric redshifts, we now repeat the SED modeling fixing the redshift to its best-fit value. The same mechanisms as discussed in $\S 4$ are still influencing the recovery of physical parameters. Given the partially random nature of the $z_{\text {phot }}$ uncertainties, it comes as no surprise that the central $68 \%$ interval broadens with respect to the SED modeling at fixed redshift. Averaged over time, the broadening is limited to $1 \%, 7 \%, 5 \%, 9 \%$, and $1 \%$ in $\Delta \log a g e_{w}$, $\Delta \log M, \Delta E(B-V)_{\text {eff }}, \Delta A_{V, \text { eff }}$, and $\Delta \log S F R$ respectively, owing to the small scatter in $z_{\text {true }}$ versus $z_{\text {phot }}$.

Any systematic part of the $z_{\text {phot }}$ uncertainty translates into additional systematic offsets in the stellar population properties. Qualitatively, when a source is mistakenly placed at higher redshift, a larger mass estimate and lower dust reddening are required to match the observed SED. Although we established that systematic offsets in $\Delta z /(1+z)$ are small (\$5.2.1), such an effect is noticeable for the most obscured $\left(A_{V}>2.5\right)$ galaxies. The slight overprediction of their redshifts translates into an additional offset in the estimated mass of $\log M_{\text {fixz }}-\log M_{\text {photz }} \sim-0.05$ dex, in the estimated reddening of $E(B-V)_{\text {fixz }}-E(B-V)_{\text {photz }} \sim 0.018 \mathrm{mag}$, and in the estimated extinction of $A_{V \text {,fixz }}-A_{V \text {,photz }} \sim 0.11$ mag. We note that, for an excellent photometric redshift accuracy as presented here, these effects are up to an order of magnitude smaller than the systematic offsets from the true stellar population properties as introduced by our poor knowledge of the star formation history, dust content and distribution, and metallicity. Furthermore, the impact of $z_{\text {phot }}$ uncertainties discussed here is specific for the particular template set used. Other template sets may introduce different redshift biases and therefore propagate into different biases when characterizing stellar populations.

\section{SUMMARY}

We analyzed the performance of a simple SED modeling procedure applied to synthetic optical-to-NIR broadband SEDs of merger simulations placed at redshifts $\mathrm{z}=1.5,1.7, \ldots, 2.9$. The synthetic SEDs span a large range of colors and shapes, and are significantly affected by dust attenuation. For all simulated galaxies, we find counterparts in the real high-redshift universe with similar SED types. First, we modeled the SEDs assuming the redshift was known. The masses, ages, $E(B-V)$, and $A_{V}$ of simulated ellipticals are very well reproduced, with an average value of $\Delta \log M=-0.02_{-0.11}^{+0.06}, \Delta \log a g e_{w}=$ $-0.03_{-0.14}^{+0.12}, \Delta E(B-V)=-0.03_{-0.07}^{+0.11}$, and $\Delta A_{V}=$ $-0.29_{-0.30}^{+0.32}$. Here the errors indicate the central $68 \%$ interval of the distribution of $\Delta$ parameter values of all the simulations (different masses, gas fractions, viewing angles) in the spheroid regime. The simulated ellipticals are correctly classified as having low $\operatorname{SFRs}(\Delta \log S F R=$ $\left.-0.23_{-0.47}^{+0.62}\right)$. In earlier, actively star-forming, phases, the scatter in recovered stellar population properties with respect to the true value increases, and larger systematic underestimates of age, mass, extinction, and SFR occur. This is particularly the case for the simulation snapshots of phases with merger-triggered star formation, where we find the following offsets and scatter (averaged over the merger regime indicated in Figure $1): \Delta \log M=-0.13_{-0.14}^{+0.10}, \Delta \log a g e_{w}=-0.12_{-0.26}^{+0.40}$,
$\Delta E(B-V)=-0.02_{-0.08}^{+0.08}, \Delta A_{V}=-0.54_{-0.46}^{+0.40}$, and $\Delta \log S F R=-0.44_{-0.31}^{+0.32}$. The SED modeling performs better on regular star-forming disks than on galaxies during the merging event. Compared to spheroids however, the results of the SED modeling on disks show a larger scatter and larger systematic underestimates: $\Delta \log M=-0.06_{-0.14}^{+0.06}, \Delta \log a g e_{w}=+0.03_{-0.42}^{+0.19}$, $\Delta E(B-V)=-0.02_{-0.07}^{+0.13}, \Delta A_{V}=-0.35_{-0.34}^{+0.29}$, and $\Delta \log S F R=-0.22_{-0.34}^{+0.23}$.

By adding the effects of dust attenuation, metallicity variations and AGN step by step to the basic intrinsic photometry, we were able to disentangle the different mechanisms at play and their impact on the estimation of the galaxy's stellar population properties. The qualitative impact on the SED modeling results by different aspects of the galaxy content is summarized in Table 1.

A mismatch between the real $\mathrm{SFH}$ and the allowed template SFHs leads to an inability to account for the difference between light-weighted and mass-weighted properties such as stellar age and mass. If the optical depth toward intrinsically bluer emitting sources is larger than to intrinsically redder stellar populations, the nett effect of the age and mass underestimate due to mismatch between true and template $\mathrm{SFH}$ is less severe. We find proof of such an increased extinction toward younger stars during the merger-triggered starburst. Applying the Calzetti et al. (2000) reddening law toward each stellar particle, we find that the overall reddening for a given $A_{V}$ is less than predicted by the Calzetti et al. (2000) law, particularly when the optical depth is uncorrelated with the intrinsic colors of the sources it is hiding. In the latter case, the effective dust vector has a shallower slope in the $U-V$ versus $V-J$ color-color diagram than the Calzetti et al. (2000) vector. In the case of larger optical depths toward young (blue) stellar populations, there is relatively more reddening in $U-V$ for a given reddening in $V-J$. Applying a MW or SMC-like attenuation law to the individual stellar particles in the simulation increases the reddening, but the effective extinction is still grayer than the Calzetti et al. (2000) law.

All other properties remaining the same, the effect of applying our SED modeling to stellar populations with sub-solar metallicities is that one would underpredict the reddening. For the young ages where such sub-solar metallicities could be expected, interpreting the light as coming from a solar-metallicity population will lead to an overestimate of the stellar age.

Finally, our SED modeling is based on purely stellar emission. During the brief period when the AGN contribution is significant, the addition of its light will make the galaxy look younger and dustier, with a larger SFR.

Although some of the biases described above could be anticipated from simpler models (see, e.g., Fig. 12 and Fig. 16), the simulations used in this paper prove very valuable in illustrating and quantifying the effects at play. Moreover, by following the formation of stars and distribution of gas and dust on a physically motivated basis, they provide insights that could not be obtained from simple toy models, such as the preferential extinction of young stars during the merger and its impact on recovering stellar population properties by SED modeling.

We next repeated our analysis adopting the best-fit 
TABle 1. QuAlitative SUMmary OF SYSTEMATIC TRENDS IN RECOVERING STELLAR POPULATION PROPERTIES.

\begin{tabular}{|c|c|c|c|c|c|}
\hline Effect & on age & on $E(B-V)$ & on $A_{V}$ & on mass & on SFR \\
\hline SFH mismatch (higher SFR than in the past) & - & + & - & - & + \\
\hline Non-uniform extinction (uncorrelated with intrinsic colors of emitters) & - & + & - & - & - \\
\hline Age-dependent extinction (more extinction toward young stars) & + & - & - & $-\mathrm{a}$ & - \\
\hline$Z<Z \odot$ & + & - & - & $++^{b}$ & 0 \\
\hline AGN & - & + & + & $+^{\mathrm{c}}$ & + \\
\hline
\end{tabular}

a The overestimated age and underestimated $A_{V}$ compete in the determination of stellar mass. Since in practice we find this effect to be most outspoken in phases where the SFH mismatch is largest, the stellar mass will effectively be underestimated.

${ }^{\mathrm{b}}$ Here too, the systematic offsets in age and $A_{V}$ have an opposite sign. From comparison of mass estimates based on the full attenuated photometry and its equivalent with all stars fixed to $Z_{\odot}$, we find the effective $\Delta \log M$ is positive.

cSimilar to (b), we compared the results from SED modeling with/out AGN contribution and find that in the median the addition of AGN light increases the mass estimate slightly.

photometric redshift estimate. Using the photometric redshift code EAZY (Brammer et al. in preparation), we obtain a median normalized absolute deviation $\sigma_{\mathrm{NMAD}}(\Delta z /(1+z))=0.031$. The random uncertainty in $z_{\text {phot }}$ boosts the scatter in the quality measures $\Delta \log M$, $\Delta \log a g e_{w}, \Delta E(B-V), \Delta A_{V}$, and $\Delta \log S F R$ by less than $10 \%$. A slight dependence of $\Delta z /(1+z)$ on extinction propagates into an additional systematic difference in the estimated stellar mass, on the $10 \%$ level for galaxies with $A_{V}>2.5$. Offsets in reddening and visual extinction $A_{V}$ are anti-correlated with $\Delta z /(1+z)$.

S. W. and T. J. C. acknowledge support from the W. M. Keck Foundation. B. E. R. gratefully acknowledges support from a Spitzer Fellowship through a NASA grant administrated by the Spitzer Science Center. We thank Natascha Förster Schreiber for useful discussions on stellar populations.

\section{REFERENCES}

Barnes, J. E.,\& Hernquist, L. 1996, ApJ, 471, 115

Barnes, J. E.,\& Hernquist, L. 1991, ApJ, 370, L65

Bell, E. F.,\& de Jong, R. S. 2001, ApJ, 550, 212

Bolzonella, M., Miralles, J.-M.,\& Pelló, R. 2000, A\&A, 363, 476

Brammer, G. B., van Dokkum, P. G.,\& Coppi, P. 2008, ApJ, 686, 1503

Bruzual, G.,\& Charlot, S. 2003, MNRAS, 344, 1000 (BC03)

Bullock, J. S., Kolatt, T. S., Sigad, Y., Somerville, R. S., Kravtsov, A. V., Klypin, A. A., Primack, J. R.,\& Dekel, A. 2001, MNRAS, 321, 559

Calzetti, D., et al. 2000, ApJ, 533, 682

Cox, T. J., Dutta, S. N., Di Matteo, T., Hernquist, L., Hopkins, P. F., Robertson, B.,\& Springel, V. 2006, ApJ, 650,791

Daddi, E., et al. 2007a, ApJ, 670, 156

Daddi, E., et al. 2007b, ApJ, 670, 173

Di Matteo, T., Springel, V.,\& Hernquist, L. 2005, Nature, 433,604

Driver, S. P., Windhorst, R. A.,\& Griffiths, R. E. 1995, ApJ, 453, 48

Edmunds, M. G. 1990, MNRAS, 246, 678

Erb, D. K., Shapley, A. E., Pettini, M., Steidel, C. C., Reddy, N. A.,\& Adelberger, K. L. 2006a, ApJ, 644, 813

Erb, D. K., Steidel, C. C., Shapley, A. E., Pettini, M., Reddy, N. A.,\& Adelberger, K. L. 2006b, ApJ, 646, 107

Erb, D. K., Steidel, C. C., Shapley, A. E., Pettini, M., Reddy, N. A.,\& Adelberger, K. L. 2006c, ApJ, 647, 128

Ferguson, H. C., Dickinson, M., Papovich, C. 2002, ApJ, 569, 65

Fioc, M.,\& Rocca-Volmerange, B. 1997, A\&A, 326, 950

Förster Schreiber, N. M., et al. 2004, ApJ, 616, 40

Förster Schreiber, N. M., et al. 2006, AJ, 131, 1891

Franx, M., Illingworth, G. D., Kelson, D. D., van Dokkum, P. G.,\& Tran, K. 1997, ApJ, 486, L75

Genzel, R., et al. 1998, ApJ, 498, 579

Glazebrook, K., Ellis, R. S., Santiago, B.,\& Griffiths, R. 1995, MNRAS, 275, L19

Grazian, A., et al. 2006a, A\&A, 449, 951
Groves, B., Dopita, M. A., Sutherland, R. S., Kewley, L. J., Fischera, J., Leitherer, C., Brandl, B.,\& van Breugel, W. 2008, ApJS, 176, 438

Hernquist, L. 1989, Nature, 340, 687

Holden, B. P., et al. 2005, ApJ, 620, 83

Holmberg, E. 1941, ApJ, 94, 385

Hopkins, P. F., Hernquist, L., Martini, P., Cox, T. J., Robertson, B., Di Matteo, T.,\& Springel, V. 2005a, ApJ, 625, L71

Hopkins, P. F., Hernquist, L., Cox, T. J., Di Matteo, T., Martini, P., Robertson, B.,\& Springel, V. 2005b, ApJ, 630, 705

Hopkins, P. F., Richards, G. T.,\& Hernquist, L. 2007, ApJ, 654,731

Jonsson, P. 2006, MNRAS, 372, 2

Jonsson, P., Cox, T. J., Primack, J. R.,\& Somerville, R. S. 2006, ApJ, 637, 255

Kriek, M., et al. 2006, ApJ, 645, 44

Kriek, M., et al. 2007, ApJ, 669, 776

Kroupa, P. 2001, MNRAS, 322, 231

Labbé, I., et al. 2003, AJ, 125, 1107

Larson, R. B.,\& Tinsley, B. M. 1978, ApJ, 219, 46

Maiolino, R., et al. 2008, A\&A, 488, 463

Maraston, C. 2005, MNRAS, 362, 799

Maraston, C., Daddi, E., Renzini, A., Cimatti, A., Dickinson, M., Papovich, C., Pasquali, A.,\& Pirzkal, N. 2006, ApJ, 652,85

Mihos, J. C.,\& Hernquist, L. 1994, ApJ, 431, L9

Mihos, J. C.,\& Hernquist, L. 1996, ApJ, 464, 641

Mo, H. J., Mao, S.,\& White, S. D. M. 1998, MNRAS, 295, 319

Noguchi, M. 1988, A\&A, 203, 259

O'Connell, R. W. 1986, in Stellar Populations, ed. C. Norman, A. Renzini,\& M. Tosi (Cambridge: Cambridge Univ. Press), 213

Panuzzo, P., Granato, G. L., Buat, V., Inoue, A. K., Silva, L., Iglesias-Páramo, J.,\& Bressan, A. 2007, MNRAS, 375, 640

Papovich, C., Dickinson, M., \& Ferguson, H. C. 2001, ApJ, 559, 620 
Papovich, C., Dickinson, M., Giavalisco, M., Conselice, C. J.,\& Ferguson, H. C. 2005, ApJ, 631, 101

Papovich, C., et al. 2006, ApJ, 640, 92

Papovich, C., et al. 2007, ApJ, 668, 45

Pei, Y. C. 1992, ApJ, 395, 130

Pettini, M., Kellogg, M., Steidel, C. C., Dickinson, M., Adelberger, K. L.,\& Giavalisco, M. 1998, ApJ, 508, 539

Pettini, M., et al. 2001, ApJ, 554, 981

Reddy, N. A., Erb, D. K., Steidel, C. C., Shapley, A. E., Adelberger, K. L,\& Pettini, M. 2005, ApJ, 633, 748

Reddy, N. A., Steidel, C. C., Fadda, D., Yan, L., Pettini, M., Shapley, A. E., Erb, D. K., \& Adelberger, K. L., ApJ, 644, 792

Richards, G. T., et al. 2006, ApJS, 166, 470

Rigopoulou, D., et al. 2006, ApJ, 648, 81

Robertson, B., Cox, T. J., Hernquist, L., Franx, M., Hopkins, P. F., Martini, P.,\& Springel, V. 2006, ApJ, 641, 21

Robertson, B., Li, Y., Cox, T. J., Hernquist, L.,\& Hopkins, P. F. 2007, ApJ, 667, 60

Rocha, M., Jonsson, P., Primack, J. R.,\& Cox, T. J. 2008, MNRAS, 383, 1281

Sanders, D. B., Soifer, B. T., Elias, J. H., Madore, B. F., Matthews, K., Neugebauer, G.,\& Scoville, N. Z. 1988a, ApJ, 325, 74

Sanders, D. B., Soifer, B. T., Elias, J. H., Neugebauer, G.,\& Matthews, K. 1988b, ApJ, 328, L35
Sanders, D. B.,\& Mirabel, I. F. 1996, ARA\&A, 34, 749

Shapley, A. E., Steidel, C. C., Adelberger, K. L., Dickinson, M., Giavalisco, M.,\& Pettini, M. 2001, ApJ, 562, 95

Shapley, A. E., Steidel, C. C., Pettini, M.,\& Adelberger, K. L. 2003, ApJ, 588, 65

Shapley, A. E., Steidel, C. C., Erb, D. K., Reddy, N. A., Adelberger, K. L., Pettini, M., Barmby, P.,\& Jiasheng, H. 2005, ApJ, 626, 698

Springel, V.,\& Hernquist, L. 2003, MNRAS, 339, 289

Springel, V., Di Matteo, T.,\& Hernquist, L. 2005a, ApJ, 620, L79

Springel, V., Di Matteo, T.,\& Hernquist, L. 2005b, MNRAS, 361,776

Toomre, A.,\& Toomre, J. 1972, ApJ, 178, 623

Toomre, A. 1977, in Evolution of Galaxies and Stellar Populations, 401, Yale Univ. Obs: New Haven

van Dokkum, P. G.,\& Stanford, S. A. 2003, ApJ, 585, 78

van Dokkum, P. G., et al. 2004, ApJ, 611, 703

Vitvitska, M., Klypin, A. A., Kravtsov, A. V., Wechsler, R. H., Primack, J. R.,\& Bullock, J. S. 2002, ApJ, 581, 799

Wuyts, S., et al. 2007, ApJ, 655, 51

Wuyts, S., et al. 2008, ApJ, 682, 985

Wuyts, S., et al. 2009b, submitted to ApJ

Zwicky, F. 1956, Ergebnisse der Exakten Naturwissenschaften, 29, 344 